\title{
NOTES ON DERIVATIONS OF MURRAY-VON NEUMANN ALGEBRAS
}

\author{
ALEKSEY BER, KARIMBERGEN KUDAYBERGENOV, AND FEDOR SUKOCHEV
}

To the memory of Richard Kadison

\begin{abstract}
Let $\mathcal{M}$ be a type $\mathrm{II}_{1}$ von Neumann factor and let $S(\mathcal{M})$ be the associated Murray-von Neumann algebra of all measurable operators affiliated to $\mathcal{M}$. We extend a result of Kadison and Liu 29] by showing that any derivation from $S(\mathcal{M})$ into an $\mathcal{M}$-bimodule $\mathcal{B} \subsetneq S(\mathcal{M})$ is trivial. In the special case, when $\mathcal{M}$ is the hyperfinite type $\mathrm{II}_{1}$-factor $\mathcal{R}$, we introduce the algebra $A D(\mathcal{R})$, a noncommutative analogue of the algebra of all almost everywhere approximately differentiable functions on $[0,1]$ and show that it is a proper subalgebra of $S(\mathcal{R})$. This algebra is strictly larger than the corresponding ring of continuous geometry introduced by von Neumann. Further, we establish that the classical approximate derivative on (classes of) Lebesgue measurable functions on $[0,1]$ admits an extension to a derivation from $A D(\mathcal{R})$ into $S(\mathcal{R})$, which fails to be spatial. Finally, we show that for a Cartan masa $\mathcal{A}$ in a hyperfinite $\mathrm{II}_{1}$-factor $\mathcal{R}$ there exists a derivation $\delta$ from $\mathcal{A}$ into $S(\mathcal{A})$ which does not admit an extension up to a derivation from $\mathcal{R}$ to $S(\mathcal{R})$.
\end{abstract}

\section{INTRODUCTION}

Let $\mathcal{A}$ be an algebra over the field of complex numbers and $\mathcal{B}$ be an $\mathcal{A}$-bimodule. A linear operator $D: \mathcal{A} \rightarrow \mathcal{B}$ is called a derivation if it satisfies the identity $D(x y)=$ $D(x) y+x D(y)$ for all $x, y \in \mathcal{A}$. Each element $a \in \mathcal{B}$ defines a linear derivation $\operatorname{ad}_{a}: D: \mathcal{A} \rightarrow \mathcal{B}$ given by $\operatorname{ad}_{a}(x)=a x-x a, x \in \mathcal{A}$. Such derivations ad $a_{a}$ are called spatial derivations. If the element $a$ implementing the derivation $\operatorname{ad}_{a}$ belongs to $\mathcal{A}$, then $\operatorname{ad}_{a}$ obviously maps $\mathcal{A}$ into itself and is called inner derivation (of the algebra $\mathcal{A}$ ).

The theory of derivations in operator algebras is an important and well studied part of the general theory of operator algebras, with applications in mathematical physics (see, e.g. [10], [41]). It is well known that every derivation of a $C^{*}$-algebra is bounded (i.e. is norm continuous), and that every derivation of a von Neumann algebra is inner. For a detailed exposition of the theory of bounded derivations we refer to the monograph of Sakai [41].

The development of a non-commutative integration theory was initiated by Segal [44, who introduced new classes of (not necessarily Banach) algebras of unbounded operators, in particular the algebra $S(\mathcal{M})$ of all measurable operators affiliated with a von Neumann algebra $\mathcal{M}$ (see next section for precise definitions).

The properties of derivations of the algebra $S(\mathcal{M})$ are far from being similar to those exhibited by derivations on von Neumann algebras. On one hand, for commutative von Neumann algebra $\mathcal{M}=L_{\infty}[0,1]$, the algebra $S(\mathcal{M})$ coincides with Lebesgue space $S[0,1]$ of all measurable complex functions on the interval $[0,1]$, and the latter algebra admits non trivial (and hence, non-inner) derivations [4, 5. On the other hand, if $\mathcal{M}$

Date: June 4, 2019.

2010 Mathematics Subject Classification. 47B47, 46L51, 46L57.

Key words and phrases. von Neumann algebra, hyperfinite factor, derivation, commutator. 
is a properly infinite von Neumann algebra, then all derivations on $S(\mathcal{M})$ are inner ([1, Theorem 2.7], [8, Theorem 4.17 and Proposition 5.3], [6, Corollary 5.1] and [7, Corollary 4.2]). These two dramatically different results indicate a special interest (and difficulty) in the case when $\mathcal{M}$ is a type $\mathrm{II}_{1}$ - von Neumann algebra, and this is precisely the case in which we are interested in this paper. In this case, $S(\mathcal{M})$ is the algebra of all operators affiliated with $\mathcal{M}$, which is sometimes referred to as the Murray-von Neumann algebra associated with $\mathcal{M}$ (see e.g. [29]). It is still unknown whether the algebra $S(\mathcal{M})$ admits non-inner derivations. To our best knowledge, the question whether every derivation on $S(\mathcal{M})$ is necessarily inner was firstly posed in [3]. A partial step towards proving that $S(\mathcal{M})$ may not admit any non-inner derivations was made by Kadison and Liu [29] who showed that any derivation from $S(\mathcal{M})$ into $\mathcal{M}$ is necessarily trivial when $\mathcal{M}$ is a von Neumann algebra of type $\mathrm{II}_{1}$. In fact, it is conjectured in [29, p.211] that $S(\mathcal{M})$ does not admit non-inner derivations in this setting. In this paper, we partially confirm this conjecture by showing that any derivation from the Murray-von Neumann algebra $S(\mathcal{M})$ associated with any type $\mathrm{II}_{1}$ von Neumann algebra $\mathcal{M}$, with values in a Calkin operator space $\mathfrak{B} \subsetneq S(\mathcal{M})$ is necessarily trivial (see Theorem 3.2 ).

The result of [29] cited above corresponds to the very special case $\mathfrak{B}=\mathcal{M}$. It is worthwhile to point out that if $\mathcal{M}$ is a type $\mathrm{II}_{1}-$ factor, then every $\mathcal{M}$-bimodule $\mathfrak{B} \subseteq S(\mathcal{M})$ is automatically a Calkin operator space. In other words in this special case our result states that every derivation from $S(\mathcal{M})$ into any $\mathcal{M}$-bimodule distinct from $S(\mathcal{M})$ is trivial (see Corollary 3.3). Our proof is based on an entirely different approach to that of [29], and appears to be of interest in its own right.

The second part of the paper is concerned with extensions of derivations initially defined on abelian subalgebras $\mathcal{A}$, of a type $\mathrm{II}_{1}$ von Neumann algebra $\mathcal{M}$. Here, we concentrate on the special case where $\mathcal{M}$ coincides with the hyperfinite type $\mathrm{II}_{1}$ factor $\mathcal{R}$, and $\mathcal{A}$ coincides with a special Cartan masa in $\mathcal{R}$, the "diagonal" subalgebra $\mathcal{D}$ of $\mathcal{R}$. The algebra $\mathcal{D}$ is $*$-isomorphic to the algebra $L_{\infty}[0,1]$, and therefore, there exists a *-subalgebra $A D(\mathcal{D}) \subset S(\mathcal{R})$, which is $*$-isomorphic to the classical $*$-subalgebra of all almost everywhere approximately differentiable function of $S[0,1]$ (see Section 4.2 for precise definitions). Next, we construct a noncommutative analogue $A D(\mathcal{R})$, generalising the algebra $A D(\mathcal{D})$ of "approximately differentiable operators" in $S(\mathcal{R})$, and show that this algebra admits a derivation, which extends the approximate derivation on $A D(\mathcal{D})$ (see Theorem 5.7). The $*$-algebra $A D(\mathcal{R})$ contains as a proper $*$-subalgebra the regular ring $C_{\infty}$ of continuous geometry for $\mathbb{C}$, constructed by J. von Neumann as a completion in the rank-metric of a sum of an increasing sequence of matrix rings over the field of complex numbers. Continuous geometry was developed by J. von Neumann in the period 1935-37 in his series of article consisting of five papers (see e.g. [38, 39]). In particular, the notion of rank-distance was firstly defined in [36] (see also [39, pp. 160-161]), and described by von Neumann as "a really significant topology" (see [39, p. 137]). This topology also plays a crucial role in our construction of the algebra $A D(\mathcal{R})$. It is of interest to observe that the properties of this topology also play an important role in our extension of the Kadison-Liu result from [29], described beforehand.

Finally, in the last section of this paper we show that there exists a derivation $\delta$ from a Cartan masa $\mathcal{A}$ of $\mathcal{R}$ with values in $S(\mathcal{A})$, which cannot be extended to a derivation from $\mathcal{R} \rightarrow S(\mathcal{R})$. This derivation $\delta$ is nothing fancy, in fact it is a twisted version of the approximate derivation on $A D(\mathcal{D})$ which fails to have an extension up to a derivation on $S(\mathcal{R})$ (see Theorem 6.1). The crucial result in this proof is [9, Theorem 1.2] (restated below as Theorem 2.2), which states that the identity of the algebra $\mathcal{M}$ can not be written as commutator $[a, b]$ with $a, b \in S(\mathcal{M})$ if one of the elements $a$ or $b$ is normal. 
The paper is organized as follows. In Section 2 we gather necessary preliminaries. Section 3 is devoted to the Kadison-Liu conjecture.

In Section 4 we prove that the largest subalgebra of $S[0,1]$ which admits a unique extension of the classical derivation $\frac{d}{d t}$ on $[0,1]$ is the algebra $A D[0,1]$ of all approximately differentiable functions.

In Section 5, for a hyperfinite factor $\mathcal{R}$ of type $\mathrm{II}_{1}$, we construct a dense (with respect to the measure topology) *-regular (in the sense von Neumann) subalgebra $A D(\mathcal{R})$ in $S(\mathcal{R})$. This algebra contains a $*$-subalgebra $*$-isomorphic to the algebra $A D[0,1]$ and can be viewed as a noncommutative analogue of approximately differentiable functions. We prove that the approximate derivative on $\partial_{A D}: A D[0,1] \rightarrow S[0,1]$ can be extended up to a derivation $\delta_{A D}: A D(\mathcal{R}) \rightarrow S(\mathcal{R})$, and that this derivation is not spatial.

In Section 6 we show that a twisted version of the approximate derivative on the algebra $S(\mathcal{D})$ cannot be extended up to a derivation on the whole algebra $S(\mathcal{R})$, and prove a similar result for an arbitrary Cartan masa $\mathcal{A}$ in the hyperfinite $\mathrm{II}_{1}$-factor $\mathcal{R}$.

Acknowledgement. The authors thank Dmitriy Zanin and Galina Levitina for useful discussions and comments on earlier versions of the present paper and Jinghao Huang and Thomas Scheckter for careful reading of the manuscript and supplying useful feedback. We also thank Kenneth Dykema for discussion of Cartan subalgebras in the hyperfinite $\mathrm{II}_{1}$-factor. Some results of Section 4 were presented by the first named author at Crimea Autumn Mathematical School KROMSH-2005.

\section{Preliminaries}

In this section we briefly list some necessary facts concerning algebras of measurable operators.

Let $H$ be a Hilbert space and let $B(H)$ be the $*$-algebra of all bounded linear operators on $H$. A von Neumann algebra $\mathcal{M}$ is a weakly closed unital *-subalgebra in $B(H)$. For details on von Neumann algebra theory, the reader is referred to [18, 31, 32, 45, 48]. General facts concerning measurable operators may be found in [35, 44] (see also [49, Chapter IX] and the forthcoming book [20]). For convenience of the reader, some of the basic definitions are recalled below.

2.1. The Murray-von Neumann algebra. A densely defined closed linear operator $x: \operatorname{dom}(x) \rightarrow H$ (here the domain $\operatorname{dom}(x)$ of $x$ is a linear subspace in $H$ ) is said to be affiliated with $\mathcal{M}$ if $y x \subset x y$ for all $y$ from the commutant $\mathcal{M}^{\prime}$ of the algebra $\mathcal{M}$.

Recall that two projections $e, f \in \mathcal{M}$ are called equivalent if there exists an element $u \in \mathcal{M}$ such that $u^{*} u=e$ and $u u^{*}=f$. A projection $p \in \mathcal{M}$ is called finite, if the conditions $q \leq p$ and $q$ is equivalent to $p$ (denoted by $p \sim q$ ) imply that $q=p$. A linear operator $x$ affiliated with $\mathcal{M}$ is called measurable with respect to $\mathcal{M}$ if $\chi_{(\lambda, \infty)}(|x|)$ is a finite projection for some $\lambda>0$. Here $\chi_{(\lambda, \infty)}(|x|)$ is the spectral projection of $|x|$ corresponding to the interval $(\lambda,+\infty)$. We denote the set of all measurable operators by $S(\mathcal{M})$. Clearly, $\mathcal{M}$ is a subset of $S(\mathcal{M})$.

Let $x, y \in S(\mathcal{M})$. It is well known that $x+y$ and $x y$ are densely-defined and preclosed operators. Moreover, the (closures of) operators $x+y, x y$ and $x^{*}$ are also in $S(\mathcal{M})$. When equipped with these operations, $S(\mathcal{M})$ becomes a unital $*$-algebra over $\mathbb{C}$ (see [19]). It is clear that $\mathcal{M}$ is a $*$-subalgebra of $S(\mathcal{M})$.

For a self-adjoint $x \in S(\mathcal{M})$ we denote by $x_{+}$(respectively, $x_{-}$) its posiitve (respectively negative part), defined by $x_{+}=\frac{x+|x|}{2}$ (respectively, $x_{-}=\frac{x-|x|}{2}$ ). We note that $x_{-}$ and $x_{+}$are orthogonal, that is $x_{-} x_{+}=0$. 
If, for example, if $\mathcal{M}$ is finite, then every operator affiliated with $\mathcal{M}$ becomes measurable. In particular, the set of all affiliated operators forms a $*$-algebra, which coincides with $S(\mathcal{M})$. Following [29, 30], in the case when von Nemaunn algebra $\mathcal{M}$ is finite, we refer to the algebra $S(\mathcal{M})$ as the Murray-von Neumann algebra associated with $\mathcal{M}$.

Let $\tau$ be a faithful normal finite trace on $\mathcal{M}$. Consider the topology $t_{\tau}$ of convergence in measure or measure topology on $S(\mathcal{M})$, which is defined by the following neighborhoods of zero:

$$
N(\varepsilon, \delta)=\left\{x \in S(\mathcal{M}): \exists e \in P(\mathcal{M}), \tau(\mathbf{1}-e) \leq \delta, x e \in \mathcal{M},\|x e\|_{\infty} \leq \varepsilon\right\}
$$

where $\varepsilon, \delta$ are positive numbers, 1 is the unit in $\mathcal{M}$ and $\|\cdot\|_{\infty}$ denotes the operator norm on $\mathcal{M}$. The algebra $S(\mathcal{M})$ equipped with the measure topology is a topological algebra.

We also recall the following result (see e.g. [40, Proposition 3.3])

Proposition 2.1. Let $\mathcal{M}$ and $\mathcal{N}$ be von Neumann algebras equipped with faithful normal finite traces. If $\alpha: \mathcal{M} \rightarrow \mathcal{N}$ is a*-isomorphisms which preserves the trace. Then, $\alpha$ extends up to a*-isomorphism of $S(\mathcal{M})$ and $S(\mathcal{N})$, which is also continuous in the measure topology.

If $m$ denotes Lebesgue measure on the interval $[0,1]$, and if we consider $L^{\infty}(m)$ as an Abelian von Neumann algebra acting via multiplication on the Hilbert space $\mathcal{H}=L^{2}(m)$, with the trace given by integration with respect to $m$, then $S\left(L^{\infty}(m)\right)$ consists of all measurable functions on $[0,1]$ which are bounded except on a set of finite measure. In other words, the algebra $S\left(L^{\infty}(m)\right)$ coincides with the space $S(0,1)$ of all a.e. finite Lebesgue measurable functions on $[0,1]$ (and we will keep the later notation for this algebra) and convergence for the measure topology coincides with the usual notion of convergence in measure.

It was established in [9] that the Heisenberg relation $[a, b]=\mathbf{1}$ does not hold in the algebra of locally measurable operators affiliated with an arbitrary infinite von Neumann algebra. In the case when the von Neumann algebra is finite, it is proved there that $[a, b] \neq \mathbf{1}$ provided that $a$ is normal. For convenience of further referencing we state it in full.

Theorem 2.2. [9, Theorem 1.2] Let $\mathcal{M}$ be a von Neumann algebra and let $a, b \in S(\mathcal{M})$.

(a) If $\mathcal{M}$ is infinite, then $[a, b] \neq \mathbf{1}$.

(b) If $\mathcal{M}$ is a finite type I algebra, then $[a, b] \neq \mathbf{1}$.

(c) If a is normal, then $[a, b] \neq \mathbf{1}$.

2.2. Regular *-algebras and regularity of the algebra $S(\mathcal{M})$. Let $\mathcal{M}$ be a von Neumann algebra with a faithful normal finite trace $\tau$ and let $S(\mathcal{M})$ be the Murrey-von Neumann algebra associated with $\mathcal{M}$.

A *-subalgebra $\mathcal{A}$ of $S(\mathcal{M})$ is said to be regular, if it is a regular ring in the sense of von Neumann, i.e., if for every $a \in \mathcal{A}$ there exists an element $b \in \mathcal{A}$ such that $a b a=a$ and $c^{*} c=0$ implies $c=0$ for all $c \in \mathcal{A}$ (see e.g. [46]).

Let $a \in S(\mathcal{M})$ and let $a=v|a|$ be the polar decomposition of $a$. Then $l(a)=v v^{*}$ and $r(a)=v^{*} v$ are left and right supports of the element $a$, respectively. The projection $s(a)=l(a) \vee r(a)$ is the support of the element $a$. It is clear that $r(a)=s(|a|)$ and $l(a)=s\left(\left|a^{*}\right|\right)$. 
Let $|a|=\int_{0}^{\infty} \lambda d e_{\lambda}$ be the spectral resolution of the element $|a| \in S(\mathcal{M})$. Since $\mathcal{M}$ is finite, there exists an element $i(|a|)=\int_{0}^{\infty} \lambda^{-1} d e_{\lambda} \in S(\mathcal{M})$. Moreover,

$$
s(i(|a|))=s(|a|),|a| i(|a|)=i(|a|)|a|=s(|a|) .
$$

Set $i(a)=i(|a|) v^{*}$. We have

$$
a i(a)=v|a| i(|a|) v^{*}=v s(|a|) v^{*}=l(a), \quad i(a) a=i(|a|) v^{*} v|a|=r(a), \text { ai }(a) a=a .
$$

Therefore $S(\mathcal{M})$ is a regular $*$-algebra. The element $i(a)$ is called a partial inverse of the element $a$, it is a unique element in $S(\mathcal{M})$, such that $i(a) l(a)=i(a)$ and $i(a) a=r(a)$ (see [46, Proposition 91]).

Let $\mathcal{A}$ be a regular $*$-subalgebra of $S(\mathcal{M})$ and let $\mathbf{1} \in \mathcal{A}$. If $a \in \mathcal{A}$, then $l(a), r(a) \in \mathcal{A}$. Indeed, by [46, Proposition 88] the left and right ideals $\mathcal{A} a$ and $a \mathcal{A}$, are generated by projections and therefore there exist projections $p$ and $q$ in $\mathcal{A}$ such that $\mathcal{A} a=\mathcal{A} q, a \mathcal{A}=$ $p \mathcal{A}$. Thus $a=b q$ for some $b \in \mathcal{A}$ and so $a q=b q q=b q=a$, and therefore $r(a) \leq q$. On the other hand, $q=c a$, hence $q r(a)=\operatorname{car}(a)=c a=q$, and so $q \leq r(a)$. We conclude that $r(a)=q \in \mathcal{A}$. Similarly, $l(a)=p \in \mathcal{A}$.

\subsection{Derivations on algebras.}

Definition 2.3. Let $\mathcal{A}, \mathcal{B}$ be $*$-subalgberas in $S(\mathcal{M})$. A derivation $\delta: \mathcal{A} \rightarrow \mathcal{B}$ is called non-expansive if $s(\delta(x)) \leq s(x)$ for all $x \in \mathcal{A}$.

We define the so-called rank metric $\rho$ on $S(\mathcal{M})$ by setting

$$
\rho(x, y)=\tau(r((x-y)))=\tau(l(x-y)), x, y \in \mathcal{A} .
$$

In fact, the rank-metric $\rho$ was firstly introduced in a general case of regular rings in [36], where it was shown it is a metric. By [13, Proposition 2.1], the algebra $S(\mathcal{M})$ equipped with the metric $\rho$ is a complete topological ring .

Proposition 2.4. Let $\mathcal{A}$ be a *-regular subalgebra of $S(\mathcal{M})$. Any derivation $\delta: \mathcal{A} \rightarrow$ $S(\mathcal{M})$ is continuous with respect to the metric $\rho$.

Proof. If $x \in \mathcal{A}$, then $l(x), r(x) \in \mathcal{A}$. We have

$$
\delta(x)=\delta(l(x) \cdot x \cdot r(x))=\delta(l(x)) x+l(x) \delta(x) r(x)+x \cdot \delta(r(x)) .
$$

Therefore, (see e.g. [39, p. 161, 3( $\eta)]$ )

$$
\begin{aligned}
\tau(r(\delta(x))) & \leq \tau(r(\delta(l(x)) x))+\tau(r(l(x) \delta(x) r(x)))+\tau(r(x \cdot \delta(r(x))))= \\
& =\tau(r(\delta(l(x)) x))+\tau(r(l(x) \delta(x) r(x)))+\tau(l(x \cdot \delta(r(x)))) \leq 3 \tau(r(x)) .
\end{aligned}
$$

Hence,

$$
\rho(\delta(a), \delta(b))=\tau(r(\delta(a-b))) \leq 3 \tau(r(a-b))=3 \rho(a, b) .
$$

This completes the proof.

\section{LACK OF NON-TRIVIAL DERIVATIONS WITH VALUES IN BIMODULES OF OPERATORS AFFILIATED WITH TYPE $\mathrm{II}_{1}$-ALGEBRAS}

In this section we consider a symmetric bimodule (or, a Calkin operator space) $\mathfrak{B} \subset$ $S(\mathcal{M})$ over an arbitrary type $\mathrm{II}_{1}$-algebra $\mathcal{M}$.

We complement the Kadison-Liu result [29] by showing that the only derivation that maps $S(\mathcal{M})$ into any such $\mathcal{M}$-bimodule $\mathfrak{B}$ is trivial provided that $\mathfrak{B} \neq S(\mathcal{M})$.

We start with collecting some technical tools. 
In this section, we assume $\mathcal{M}$ is an atomless von Neumann algebra with a faithful, normal, normalized trace $\tau$. For every $x \in \mathcal{M}$, the generalised singular value function $\mu(x)$, denoted $t \rightarrow \mu(t, x)$ for $t \in[0,1]$, is defined by the formula (see, e.g., [23], [33])

$$
\mu(t, x)=\inf \left\{\|x p\|_{\infty}: p \in P(\mathcal{M}), \tau(1-p) \leq t\right\} .
$$

For a self-adjoint element $b \in S(\mathcal{M})$, let $\lambda(b)=\lambda(\cdot, b)$ be the eigenvalue function of $b$ (also known as the spectral scale, see [2], [22] and [27]) defined by

$$
\lambda(t, b)= \begin{cases}\mu\left(t, b_{+}\right), & 0<t<\tau\left(\operatorname{supp}\left(b_{+}\right)\right) \\ \left.\lim _{\varepsilon \rightarrow 0^{+}} \lambda(1-t)-\varepsilon, b_{-}\right), & \tau\left(\operatorname{supp}\left(b_{+}\right)\right) \leq t<1 .\end{cases}
$$

Assume that $\mathcal{M}=L_{\infty}(0,1)$ and $\tau(f)=\int_{0}^{1} f d m, f \in \mathcal{M}$, where $m$ is the Lebesgue measure on $(0,1)$. In this case, $S(\mathcal{M})$ consists of all complex-valued Lebesgue measurable functions $f$ on $(0,1)$, that is $S(\mathcal{M})=S(0,1)$ [23, 33]. In this setting, for every $f \in S(0,1)$ (respectively, for every real-valued $f \in S(0,1)$ ) the function $\mu(f)$ coincides with the right-continuous equimeasurable nonincreasing rearrangement of $|f|$ (see e.g. [27]):

$$
\mu(t ; f)=\inf \{s \in \mathbb{R}: m(\{x \in X:|f(x)|>s\}) \leqslant t\}, \quad t \in[0,1)
$$

(respectively,

$$
\lambda(t ; f)=\inf \{s \in \mathbb{R}: m(\{x \in X: f(x)>s\}) \leqslant t\}, \quad t \in[0,1) .)
$$

A linear subspace $\mathcal{B}$ of $S(\mathcal{M})$ is called a Calkin operator space if $x \in \mathcal{B}$ whenever $x \in S(\mathcal{M})$ and $\mu(x) \leq \mu(y)$ for some $y \in \mathcal{B}$. A Calkin function space is the term reserved for a Calkin operator space when $\mathcal{M}=L_{\infty}(0,1)$ [33, Definition 2.4.1]. If $\mathcal{B} \subseteq S(\mathcal{M})$ is a Calkin operator space, then the set $B \subset S(0,1)$ defined by

$$
B=\{x \in S(0,1): \exists z \in \mathcal{B} \text { such that } \mu(x)=\mu(z)\}
$$

is a Calkin function space. Vice versa, if $B$ is Calkin function space, then

$$
\mathcal{B}=\{x \in S(\mathcal{M}): \exists z \in B \text { such that } \mu(x)=\mu(z)\}
$$

is a Calkin operator space. This provides a canonical bijection between Calkin operator spaces and Calkin function spaces. For this results we refer the reader to [33, Theorem 2.4.4]. We claim that every non-empty Calkin operator space $\mathcal{B} \subseteq S(\mathcal{M})$ contains $\mathcal{M}$. Indeed, in view of the above, it is sufficient to prove that $L_{\infty}(0,1) \subseteq B$. Since $B \neq \emptyset$, there exists $0 \neq x_{0} \in B$. Then for some $\epsilon>0$ and for some measurable set $e \subset(0,1)$ of positive measure we have $|x| \geq \epsilon \chi_{e}$. This implies that $\chi_{e} \in B$ and so, $\chi_{[0, m(e))} \in B$. The latter, implies that $\chi_{[m(e), 2 m(e))} \in B$ and repeating this argument, we infer that $\chi_{[0,1)} \in B$. This implies the claim.

The following proposition extends [15, Proposition 3.0.3] (see also [16, Proposition 1.8] and [11, 12]). The proof follows [12] and is given here for convenience of the reader.

Proposition 3.1. If $x=x^{*} \in S(\mathcal{M}, \tau)$, then there exists an atomless commutative weakly closed $*$-subalgebra $\mathcal{N}$ in $\mathcal{M}$ containing the spectral family of the operator $x$, and $a *$-isomorphism $V$ acting from $S(\mathcal{N}, \tau)$ onto $S([0, \tau(s(x))), m)$ such that $V(x)=\lambda(x)$ and $\lambda(V(f))=\lambda(f)$ for every $f=f^{*} \in S(\mathcal{N}, \tau)$.

Proof. Let $\nabla_{0}$ be a countable Boolean subalgebra in $P(\mathcal{M})$ which contains all spectral projections $E^{x}(r, \infty)$ and $E^{x}(-\infty, r)$ of $x$, where $r$ is a rational number. Let $\nabla$ be the closure of $\nabla_{0}$ in the measure topology. Then, $\nabla$ is a complete Boolean subalgebra in $P(\mathcal{M})$ and the least upper bound in $\nabla$ for any subset $A \subset \nabla$ coincides with the least upper bounded of $\mathcal{A}$ in $P(\mathcal{M})$. Such subalgebra are also called regular. 
Let $\triangle$ be the set of all atoms in $\nabla$ and $\triangle \neq 0$. Since $P(\mathcal{M})$ is a non-atomic Boolean algebra, for every $q \in \triangle$, there exists a commutative non-atomic regular Boolean subalgebra $\nabla_{q}$ of $P\left(\mathcal{M}_{q}\right)$ which is separable in the measure topology.

Let $B$ be the set of all $e \in P(\mathcal{M})$ for which $e(1-\sup \triangle) \in \nabla$ and $e q \in \nabla_{q}$ for any $q \in \triangle$. It is clear that $B$ is a complete regular non-atomic and separable (with respect to the measure topology) Boolean subalgebra in $P(\mathcal{M})$ which contains all the spectral projections of $x$. Hence, there exists an isomorphism $\phi$ from $B$ on the Boolean algebra $P\left(L_{\infty}(0, \tau(s(x)))\right)$ such that $m(\phi(e))=\tau(e)$ for all $e \in B$ [26].

Let us denote by $\mathcal{N}$ the weak closure of the $*$-algebra generated by $B$, which is a non-atomic commutative von Neumann subalgebra of $\mathcal{M}$.

By Proposition [2.1, the isomorphism $\phi$ may be extended up to the $*$-isomorphism $V$ from $S(\mathcal{N}, \tau)$ onto $S(0, \tau(s(x)))$ and, in addition, $V(\mathcal{N})=L_{\infty}(0, \tau(s(x))), \lambda(t, x)=$ $\lambda(t, V x)$ for all $t>0, x \in S(\mathcal{M}, \tau)$.

The following result extends [29, Corollary 13].

Theorem 3.2. Let $\mathcal{M}$ be a type $I_{1}$ von Neumann algebra and let $\mathcal{B} \subsetneq S(\mathcal{M})$ be a Calkin operator space. Then any derivation $\delta: S(\mathcal{M}) \longrightarrow S(\mathcal{M})$, such that $\delta(S(\mathcal{M})) \subset \mathcal{B}$ vanishes.

Proof. Since $\delta(x) \in \mathcal{B}$ for all $x \in S(\mathcal{M})$ and $\mathcal{B}$ is closed with respect to conjugation, it follows that that $\delta(x)^{*} \in \mathcal{B}$ for all $x \in S(\mathcal{M})$. Therefore, $\frac{\delta(x)+\delta\left(x^{*}\right)^{*}}{2} \in \mathcal{B}$ and $\frac{\delta(x)-\delta\left(x^{*}\right)^{*}}{2 i} \in \mathcal{B}$ for all $x \in S(\mathcal{M})$. Thus, replacing $\delta$ with $\frac{\delta+\delta^{*}}{2}$, where $\delta^{*}(x)=$ $\delta\left(x^{*}\right)^{*}, x \in S(\mathcal{M})$, without loss of generality, we can assume that $\delta=\delta^{*}$, that is, $\delta(x)^{*}=\delta\left(x^{*}\right)$ for all $x \in S(\mathcal{M})$.

Assume that $\delta \neq 0$. If $\left.\delta\right|_{\mathcal{M}}=0$, then due to $\rho$-continuity of $\delta$ (see Proposition 2.4) and $\rho$-density of $\mathcal{M}$ in $S(\mathcal{M})$, we obtain that $\delta=0$, which contradicts with the assumption $\delta \neq 0$. So, if $\delta \neq 0$, then there exists a self-adjoint element $a \in \mathcal{M}$ such that $\delta(a) \neq 0$.

The operator $\delta(a)$ is self-adjoint, and so, by Proposition 3.1, there exists an atomless commutative weakly closed $*$-subalgebra $\mathcal{N}$ in $\mathcal{M}$ and a $*$-isomorphism $V$ acting from $S(\mathcal{N}, \tau)$ onto $S([0, \tau(s(\delta(a)))), m)$ such that $V(\delta(a))=\lambda(\delta(a))$. Setting $\pi=V^{-1}$ and

$$
y=\lambda(\delta(a)),
$$

we have

$$
\pi(y)=\delta(a) \quad \text { and } \quad \pi\left(\chi_{[0, \tau(s(\delta(a))))}\right)=s(\delta(a)),
$$

because by construction of $V$ the support of $y$ is $[0, \tau(s(\delta(a))))$. In particular, $y$ is invertible in $S[0, \tau(s(\delta(a))))$. We claim that the assumptions $\mathcal{B} \neq S(\mathcal{M})$ and $\mathcal{B}$ is a Calkin operator space, imply that there exists $x \in S[0, \tau(s(\delta(a))))$ such that $\pi(x) \notin \mathcal{B}$. Indeed, let us consider the function Calkin space $B$ introduced above. Since $\mathcal{B} \neq S(\mathcal{M})$, it follows that $B \neq S(0,1)$. Now, we simply take any $z=\mu(z) \in S(0,1)$ such that $z \notin B$ and set $x=\mu(z) \cdot \chi_{[0, \tau(s(\delta(a)))}$. Observe that $x \notin B$. Indeed, by the claim preceding Proposition 3.1, we know that the bounded function $z-x \in B$ and therefore $x \notin B$. The fact that $\pi(x) \notin \mathcal{B}$ now follows immediately from the fact that $\mu(\pi(x))=\mu(x)$ established in [40, Proposition 3.3(ii)]. We shall now finish the proof. Take $b=\pi\left(y^{-1} x\right)$. Then

$$
\delta(a) b=\pi(y) b=\pi(y) \pi\left(y^{-1} x\right)=\pi(x) .
$$

Computing $\pi(x)=\delta(a) b=\delta(a b)-a \delta(b) \in \mathcal{B}$, we arrive at the contradiction. 
Recall that a linear subspace $\mathcal{J}$ of $S(\mathcal{M})$ is called an operator bimodule on $\mathcal{M}$ if $A B, B A \in \mathcal{J}$ whenever $A \in \mathcal{J}$ and $B \in \mathcal{M}$ [33, Definition 2.4.5]. If $\mathcal{M}$ is a finite factor, then every operator bimodule is a Calkin operator space [33, Lemma 2.4.6].

Corollary 3.3. Let $\mathcal{M}$ be a II $I_{1}$-factor and let $\mathcal{B} \subsetneq S(\mathcal{M})$ be a $\mathcal{M}$-bimodule. If $D$ : $S(\mathcal{M}) \rightarrow S(\mathcal{M})$ is a derivation and $D(S(\mathcal{M})) \subset \mathcal{B}$, then $D \equiv 0$.

\section{Approximate Derivative as a Unique extension of the Classical DERIVATION $\frac{d}{d t}$}

Let $\mathcal{A}=S[0,1]$ be the *-algebra of all classes of Lebesgue measurable functions on $[0,1]$ (as usual, the quotient is taken with respect to the relation "equal almost everywhere"), which is the Murray-von Neumann algebra associated with the finite von Neumann algebra $L_{\infty}[0,1]$ of all (classes of) bounded functions on $[0,1]$. Consider the algebra $D[0,1]$ of (classes of) differentiable functions that is having almost everywhere finite derivation on $[0,1]$. Obviously, $D[0,1]$ is a $*$-subalgebra of $S[0,1]$.

We denote by $\lambda$ the Lebesgue measure on $[0,1]$. Sometimes, we denote by $[f]$ the class in $S[0,1]$, containing a measurable function $f$ on $[0,1]$. However, frequently we do not distinguish between $f$ and $[f]$.

In this section we show that the classical derivation $\frac{d}{d t}$ on the algebra $D[0,1]$ of all differentiable functions on $[0,1]$ (which is correctly defined, see Proposition 4.1 below) extends uniquely to the algebra of all approximately differentiable functions that is having almost everywhere finite approximative derivation. Furthermore, this algebra is the largest $*$-algebra in $S[0,1]$ which admits a unique extension of this derivation.

4.1. The classical derivation $\frac{d}{d t}$ on $D[0,1]$. We start by showing that the classical derivation $\frac{d}{d t}$ is well-defined on the $*$-algebra $D[0,1]$.

Note that for any differentiable function $f \in S[0,1]$, the derivative $f^{\prime}$ is a measurable function as the pointwise limit of a sequence on measurable functions.

Proposition 4.1. Let $f$ and $g$ be almost everywhere differentiable functions on $[0,1]$ and $f=g$ almost everywhere. Then the set of all points in which $f$ and $g$ simultaneously have finite derivative has full measure, and the derivatives $f^{\prime}$ and $g^{\prime}$ are measurable and equal almost everywhere.

Proof. Let $A$ be the set of all points $t \in[0,1]$ such that $f(t)=g(t)$ and both derivatives $f^{\prime}, g^{\prime}$ exist and finite. By [24, Theorem 3.1.4], $f^{\prime}$ and $g^{\prime}$ are measurable on $A$. The function $h=f-g$ has everywhere defined derivative $f^{\prime}-g^{\prime}$ on $A$. Since $h(t)=0$ for all $t \in A$, the equality $h^{\prime}(t)=0$ holds on $A$. The proof is complete since the latter set has full measure.

Proposition 4.1 allows us to correctly define the classical derivation $\partial: D[0,1] \rightarrow$ $S[0,1]$.

Definition 4.2. We define the derivation $\partial: D[0,1] \rightarrow S[0,1]$ by setting

$$
\partial([f])=\left[f^{\prime}\right], \quad[f] \in D[0,1] .
$$

The following proposition establishes that the classical derivation $\partial$ on the algebra $D[0,1]$ is non-expansive (see Definition [2.3). In particular, results of [5] are applicable to $\partial$.

Proposition 4.3. The derivation $\partial: D[0,1] \rightarrow S[0,1]$ is non-expansive. 
Proof. Let $f$ be almost everywhere differentiable on $[0,1]$ and suppose that the set $N(f):=\{t \in[0,1]: f(t)=0\}$ has non-zero measure. If $t$ is a density point of $N(f)$, and at this point there is a derivative of $f$, then we have $f^{\prime}(t)=0$. Thus $N(f)$ is a subset of the set $N\left(f^{\prime}\right):=\left\{t \in[0,1]: f^{\prime}(t)=0\right\}$. This means that $s(\partial(f))=s\left(f^{\prime}\right) \leq s(f)$. The proof is complete.

Proposition 4.4. Let $E$ be the fat Cantor set in $[0,1]$ (also known, as Smith-VolterraCantor set). Then the characteristic function $\chi_{E} \notin D[0,1]$. In particular, the algebra $D[0,1]$ does not contain all projections from $S[0,1]$.

Proof. The set $E$ is a closed nowhere dense subset of $[0,1]$ with $\lambda(E)>0$. Denote by $F$ the set of all points from $E$, which are density points for $E$. We have that $\mu(F)=\mu(E)>0$ (due to Lebesgue density theorem). Since the set $E$ is nowhere dense, it follows that in every neighbourhood of a point $t \in F$ there exist points, which do not belong to $E$. It means that finite derivative $\left(\chi_{E}\right)^{\prime}(t)$ does not exist at any point $t \in F$. Consequently, $\left[\chi_{E}\right] \notin D[a, b]$, as required.

4.2. The $*$-algebra $A D[0,1]$ of approximately differentiable functions. We recall firstly the concept of approximately differentiable functions.

Consider a Lebesgue measurable set $E \subset \mathbb{R}$, a measurable function $f: E \rightarrow \mathbb{R}$ and a point $t_{0} \in E$, where $E$ has Lebesgue density equal to 1 . If the approximate limit

$$
f_{a p}^{\prime}\left(t_{0}\right):=\operatorname{ap}-\lim _{t \rightarrow t_{0}} \frac{f(t)-f\left(t_{0}\right)}{t-t_{0}}
$$

exists and it is finite, then it is called approximate derivative of the function $f$ at $t_{0}$ and the function is called approximately differentiable at $t_{0}$ (see [42] for the details).

Note that by Lebesgue density theorem, for any measurable subset $A$ of $[0,1]$ almost every point is Lebesgue density point of $A$. Therefore, the following definition makes sense.

Definition 4.5. Let $A D[0,1]$ be the set of all classes $[f] \in S[0,1]$, for which $f$ is approximately differentiable almost everywhere.

Since a density point of two subsets $E$ and $F$ is a density point of the intersection $E \cap F$, it follows that the sum and product of two approximately differentiable functions is again approximately differentiable. Therefore, $A D[0,1]$ is a $*$-subalgebra of $S[0,1]$.

Proposition 4.6. The *-algebra $A D[0,1]$ is a regular proper *-subalgebra of $S[0,1]$ containing $D[0,1]$ and all projections from $L_{\infty}[0,1]$.

Proof. Let $f$ be a representative of $[f] \in A D[0,1]$. Then $f_{a p}^{\prime}$ is a measurable function and the function $g$ on $[0,1]$ defined as

$$
g(t)= \begin{cases}\frac{1}{f(t)}, & \text { if } f(t) \neq 0 \\ 0, & \text { if } f(t)=0\end{cases}
$$

is also approximately differentiable almost everywhere in $[0,1]$. Hence, $g \in A D[0,1]$ and $f g f=f$. Thus, the algebra $A D[0,1]$ is regular.

Since any differentiable function is approximately differentiable, it follows that $D[0,1] \subset A D[0,1]$.

Let us show that $A D[0,1]$ contains all projections from $L_{\infty}[0,1]$. Indeed, take a measurable subset $A$ in $[0,1]$. Consider a subset $A_{0} \subseteq A$ the set of all points of density of $A$. By Lebesgue's density theorem we know that Lebesgue measure of the set $A \backslash A_{0}$ vanishes. Since the characteristic function $\chi_{A_{0}}$ has an approximate derivative equal 
to zero almost everywhere in $A_{0}$, it follows that the class containing the function $\chi_{A}$ belong to $A D[0,1]$. Hence $A D[0,1]$ contains all projections from $L_{\infty}[0,1]$.

Finally, to show that $A D[0,1]$ is a proper subalgebra of $S[0,1]$, let $f$ be a measurable function $[0,1]$ which is not approximately differentiable almost everywhere on $[0,1]$ (such function exists as shown in [42, Chap. IX, §11]). Let $f \sim g$ and let $g$ has an approximate derivative at point $t_{0}$. Let $A \subseteq[0,1]$ be a measurable subset with $\lambda(A)>0$ such that $t_{0} \in A$. Since $f \sim g$, it follows that the set $A$ and its subset $A \cap\{t \in[0,1]: f(t)=g(t)\}$ have same measure and therefore their sets of all density points also coincide. Therefore a function $f$ also has a finite approximate derivative at point $t_{0}$. Hence, the function $g$ does not admit a finite approximate derivative almost everywhere on $[0,1]$. Due to the arbitrary choice of $g \sim f$, we conclude that $[f] \notin A D[0,1]$. The proof is complete.

We need the following characterization of the algebra $A D[0,1]$.

Proposition 4.7. The *-subalgebra $A D[0,1]$ coincides with the set of all functions of the form

$$
\sum_{n=1}^{\infty} \chi_{A_{n}} g_{n}
$$

with $A_{n} \cap A_{k}=\emptyset, n \neq k, \lambda\left(\bigcup A_{n}\right)=1$ and $g_{n} \in C^{1}[0,1], n \in \mathbb{N}$.

Proof. We prove firstly that any function of the form (4.1) is approximately differentiable almost everywhere.

For each $i$ denote by $\widetilde{A_{i}}$ the set of all density points $t$ of $A_{i}$ such that there is a finite derivative $g_{i}^{\prime}(t)$. Since $g_{i}$ is almost everywhere differentiable, due to Lebesgue density theorem [42, Theorem 10.2], we obtain that $\lambda\left(A_{i} \triangle \widetilde{A_{i}}\right)=0$. Then at each point $t \in A_{i} \cap \widetilde{A_{i}}$ a function $f$ has an approximate derivative equal to $g_{i}^{\prime}(t)$. Therefore $f \in A D[0,1]$.

The converse inclusion follows from the fact that any approximately differentiable function is continuously differentiable outside of a set of arbitrarily small measure [24, Theorem 3.1.16].

Recall (see Section 2.3) that the complete metric $\rho$ on $S[0,1]$ is defined by

$$
\rho(x, y)=\lambda(s(x-y)), \quad x, y \in S[0,1] .
$$

We say that a $*$-subalgebra $\mathcal{A} \subset S[0,1]$ is topologically closed if $(\mathcal{A}, \rho)$ is a complete metric space.

Proposition 4.8. The *-algebra $A D[0,1]$ is the smallest regular, topologically closed *-subalgebra of $S[0,1]$ containing $D[0,1]$ and all projections from $S[0,1]$.

Proof. By Proposition 4.6 the algebra $A D[0,1]$ is regular and contains $D[0,1]$ and all projections from $S[0,1]$. We now show that $A D[0,1]$ is topologically closed. Let $[f]$ be a $\rho$-limit point of $A D[0,1]$. Then for each $n \in \mathbb{N}$ there is a measurable subset $A_{n}$ in $s(f)$ such that $\lambda\left(s(f) \backslash A_{n}\right)<1 / n$ and $\left[f \chi_{A_{n}}\right] \in A D[0,1]$. Hence, $f=\sum_{n=1}^{\infty} f_{n}$, where $f_{1}=f \chi_{A_{1}}, f_{n}=f \chi_{A_{n} \backslash \cup_{k=1}^{n-1} A_{k}}$ for $n>1$. By Proposition 4.7, every $f_{n}$ is of the form (4.1), and therefore, $f$ is also of the form (4.1). Using again Proposition 4.7 we conclude that $[f] \in A D[0,1]$, that is the algebra $A D[0,1]$ is topologically closed.

Let $\mathcal{A} \subset A D[0,1]$ be a regular, topologically closed $*$-subalgebra of $S[0,1]$, which contains $D[0,1]$ and all projections from $S[0,1]$. Let $f \in A D[0,1]$. By Proposition 4.7, 
$f$ has the form

$$
f=\sum_{n=1}^{\infty} \chi_{A_{n}} g_{n}
$$

for some with $A_{n} \cap A_{k}=\emptyset, n \neq k, \lambda\left(\bigcup A_{n}\right)=1$ and $g_{n} \in C^{1}[0,1], n \in \mathbb{N}$. Note that the partial sums $\sum_{n=1}^{k} \chi_{A_{n}} g_{n}, k \in \mathbb{N}$ are contained in $\mathcal{A}$. By [5, Proposition 2.7] the series $f=\sum_{n=1}^{\infty} \chi_{A_{n}} g_{n}$, converges with respect the metric $\rho$. Therefore, $f \in \mathcal{A}$, that is $\mathcal{A}=A D[0,1]$.

Definition 4.9. Let $\mathcal{A}$ be a $*$-subalgebra of $S[0,1]$. Denote by $\mathcal{A}[x]$ the $*$-algebra of all polynomials with coefficients from $\mathcal{A}$. An element $a \in S[0,1]$ is said to be integral with respect to $\mathcal{A}$, if there exists a unitary polynomial $p \in \mathcal{A}[x]$ such that $p(a)=0$. The algebra $\mathcal{A}$ is said to be integrally closed if it contains all elements from $S[0,1]$, which are integral with respect to $\mathcal{A}$.

Proposition 4.10. The algebra $A D[0,1]$ is integrally closed.

Proof. Let us firstly consider the special case when $[f] \in S[0,1]$ is integral with respect to $D[0,1]$, i.e., $f$ is a root of unitary polynomial $p(x)=x^{m}+a_{1} x^{m-1}+\ldots+a_{m}$ with coefficients $a_{k}, 1 \leq k \leq m$ being given by almost everywhere differentiable functions on $[0,1]$. In addition, we can assume that for almost all $t \in[0,1]$ the number $f(t)$ is a simple root of a complex polynomial $p(\xi)=\xi^{m}+a_{1}(t) \xi^{m-1}+\ldots+a_{m}(t)$ (see [5, Proposition 3.3]).

If $m=1$, then $f$ is a root of unitary polynomial $p(x)=x+a_{1}$, and therefore $f=-a_{1}$ is almost everywhere differentiable on $[0,1]$. Therefore, we assume that $m \geq 2$. By the choice of $p$, for almost all points $t \in[0,1]$ the scalar $f(t)$ is a simple root of a polynomial $p(\xi)$. Let us fix one of such points $t_{0} \in[0,1]$ and set $z_{0}=\left(a_{1}\left(t_{0}\right), \ldots, a_{m}\left(t_{0}\right), f\left(t_{0}\right)\right) \in$ $\mathbb{C}^{m+1}$. Consider the function $F$ on $\mathbb{C}^{m+1}$ defined by

$$
F\left(\xi_{1}, \ldots, \xi_{m}, y\right)=y^{m}+\xi_{1} y^{m-1}+\ldots+\xi_{m}
$$

It is differentiable on $\mathbb{C}^{m+1}$, moreover,

$$
F\left(z_{0}\right)=0 \text { and } F_{y}^{\prime}=m y^{m-1}+(m-1) \xi_{1} y^{m-2}+\ldots+\xi_{m-1} .
$$

Note that $F_{y}^{\prime}\left(z_{0}\right) \neq 0$, because by our choose of $t_{0}$, the number $f\left(t_{0}\right)$ is a simple root of $p(\xi)$. Since $F_{y}^{\prime}$ is continuous, there is a neighbourhood $V\left(z_{0}\right) \subset \mathbb{C}^{m+1}$ of $z_{0}$ such that for any $z \in V\left(z_{0}\right)$ we have $F_{y}^{\prime}(z) \neq 0$. Moreover, all other partial derivative is $F_{\xi_{k}}^{\prime}=y^{m-k}$ are continuous. Hence, $F$ satisfy all conditions of the implicit function theorem (see e.g. [34, Page 315]). Thus by implicit function theorem there exists a neighbourhood $W \subset \mathbb{C}^{m}$ of $\left(a_{1}\left(t_{0}\right), \ldots, a_{m}\left(t_{0}\right)\right)$ such that $W \subset \pi\left(V\left(z_{0}\right)\right)$ (here a projection $\pi: \mathbb{C}^{m+1} \rightarrow \mathbb{C}^{m}$ defined as $\left.\pi\left(\xi_{1}, \ldots, \xi_{m+1}\right)=\left(\xi_{1}, \ldots, \xi_{m}\right)\right)$ and there is a unique differentiable function $G: W \rightarrow \mathbb{C}$ such that

$$
G\left(a_{1}\left(t_{0}\right), \ldots, a_{m}\left(t_{0}\right)\right)=f\left(t_{0}\right) \text { and } F(w, G(w))=0 \text { for all } w \in W .
$$

Take $\varepsilon>0$ such that $\left(a_{1}(t), \ldots, a_{m}(t)\right) \in W$ for almost all $t \in\left(t_{0}-\varepsilon, t_{0}+\varepsilon\right)$. Then $g(t)=G\left(a_{1}(t), \ldots, a_{m}(t)\right)$ is almost everywhere differentiable on $\left(t_{0}-\varepsilon, t_{0}+\varepsilon\right)$. Since $F(w, G(w))=0$ for all $w \in W$ and $\left(a_{1}(t), \ldots, a_{m}(t)\right) \in W$ for almost all $t \in\left(t_{0}-\varepsilon, t_{0}+\right.$ $\varepsilon)$, it follows that $p\left(g(t)=0\right.$ for almost all $t \in\left(t_{0}-\varepsilon, t_{0}+\varepsilon\right)$. Thus $\chi_{B} g$ is a root of the polynomial $p$, where $B=\left(t_{0}-\varepsilon, t_{0}+\varepsilon\right)$. Since $f$ is also root of the polynomial $p$, 
it follows that $\chi_{B} f$ is a root of the polynomial $\frac{p(x)-p(g(t))}{x-g(t)}$ whose degree is strictly less than $m$. Hence,

$$
\lambda\left(\{t: f(t)=g(t)\} \cap\left(t_{0}-\varepsilon, t_{0}+\varepsilon\right)\right)=0,
$$

that is, $f(t)$ and $g(t)$ coincide almost everywhere in $\left(t_{0}-\varepsilon, t_{0}+\varepsilon\right)$. This means that $f$ is an almost everywhere differentiable function.

Now we shall consider the general case when $[f] \in S[0,1]$ is integral with respect to $A D[0,1]$. It follows from Proposition 4.7 that $f$ is a root of a unitary polynomial $p(x)=x^{m}+a_{1} x^{m-1}+\ldots+a_{m}$ with coefficients $a_{i}(1 \leq i \leq m)$, where all $a_{i}$ is of the form (4.1). Let $a_{i}=\sum_{n=1}^{\infty} \chi_{A_{i, n}} g_{i, n}$, where $A_{i, n} \cap A_{i, k}=\emptyset$ for $n \neq k, \lambda\left(\bigcup_{n} A_{i, n}\right)=1$ and $g_{i, n}$ is an almost everywhere differentiable function on $[0,1]$ for all $n, i \in \mathbb{N}$. Further consider a partition of $[0,1]$ consisting from subsets of the form $\bigcap_{i=1}^{m} A_{i, n_{i}}, n_{1}, \ldots, n_{m} \in \mathbb{N}$. For each $\bigcap_{i=1}^{m} A_{i, n_{i}}$ with a non zero Lebesgue measure there exists a sequence of disjoint intervals $\left\{\left[a_{j}, b_{j}\right]: j \in J\right\}$ in $[0,1]$ (depending on $\left.\bigcap_{i=1}^{m} A_{i, n_{i}}\right)$ such that $\lambda\left(\bigcap_{i=1}^{m} A_{i, n_{i}} \triangle \bigcup_{j}\left[a_{j}, b_{j}\right]\right)=0$. Then $\chi_{\left[a_{j}, b_{j}\right]} f(t)$ is a root of $p_{\left[a_{j}, b_{j}\right]}(x)=x^{m}+\chi_{\left[a_{j}, b_{j}\right]} a_{1} x^{m-1}+\ldots+\chi_{\left[a_{j}, b_{j}\right]} a_{m}$, where all coefficients $\chi_{\left[a_{j}, b_{j}\right]} a_{i}=\chi_{\left[a_{j}, b_{j}\right]} g_{i, n(j)}$ are almost everywhere differentiable on $\left[a_{j}, b_{j}\right]$. Considering instead $[0,1]$ intervals $\left[a_{j}, b_{j}\right]$ in the previously treated special case, we obtain that a function $f$ coincide with an almost differentiable function on $\left[a_{j}, b_{j}\right]$. Using again Proposition 4.7, we conclude that $[f] \in A D[0,1]$, that is the algebra $A D[0,1]$ is integrally closed, as required.

Propositions 4.8 and 4.10 imply the following

Corollary 4.11. The algebra $A D[0,1]$ is the smallest regular, topologically and integrally closed $*$-subalgebra of $S[0,1]$, which contains $D[0,1]$ and all projections from $S[0,1]$.

For a $*$-subalgebra $\mathcal{B}$ in $S[0,1]$ denote by $M_{n}(\mathcal{B})$ the $*$-algebra of all $n \times n$ matrices over $\mathcal{B}$.

The next Proposition will be used in the following Section.

Proposition 4.12. Let $h=h(t)$ be a measurable function on $[0,1]$ which is nowhere approximate differentiable. Then for any matrix $A=\left(a_{i, j}\right)_{i, j=1}^{n} \in M_{n}(A D[0,1])$ a matrix $h E-A$ is not invertible in $M_{n}(S[0,1])$, where $E$ is the unit matrix in $M_{n}(S[0,1])$.

Proof. By [17, Proposition 1.3.9 (ii)] the matrix $h E-A$ is invertible if and only if its $S[0,1]$-valued determinant $\operatorname{det}(h E-A)$ is invertible in $S[0,1]$. Suppose that $\operatorname{det}(h E-A)$ is not invertible. This means that there exists a measurable subset $X$ in $[0,1]$ with a non zero measure such that $\chi_{X} \operatorname{det}(h E-A)=0$. Note that $\chi_{X} \operatorname{det}(h E-A)$ is a unitary polynomial over $A D[0,1]$ of variable $\chi_{X} h$. By Proposition 4.10, the algebra $A D[0,1]$ is integrally closed, and therefore $\chi_{X} h \in A D[0,1]$. Hence $h$ is approximately differentiable on density points $X$, which contradicts with the choice of $h$.

4.3. Approximate derivative as the largest extension of the classical derivative. In this subsection we show that $A D[0,1]$ is the largest *-subalgebra of $S[0,1]$, which admits unique extension of the classical derivation $\partial: D[0,1] \rightarrow S[0,1]$ (see Definition 4.2). We start with the following 
Proposition 4.13. Let $A D[0,1]$ be the $*$-subalgebra of $S[0,1]$ of all approximately differentiable functions. There exists a unique non-expansive derivation $\partial_{A D}: A D[0,1] \rightarrow$ $S[0,1]$, which extends the classical derivation $\partial: D[0,1] \rightarrow S[0,1]$.

Proof. By Proposition 4.3 the derivation $\partial: D[0,1] \rightarrow S[0,1]$ is nonexpansive. By [5, Proposition 2.4, Proposition 2.5], there exists a unique non-expansive extension $\delta$ of the derivation $\partial$ up to the least regular subalgebra of $S[0,1]$ generated by $D[0,1]$ and all projections from $S[0,1]$. By [5, Proposition 2.6] the derivation $\delta$ can be extended uniquely up to a derivation on the least topologically closed regular subalgebra of $S[0,1]$ containing $D[0,1]$ and all projections from $S[0,1]$. However, by Proposition 4.8 above the latter algebra coincides with $A D[0,1]$. Thus, there exists a unique extension $\partial_{A D}$ : $A D[0,1] \rightarrow S[0,1]$ of the derivation $\partial: D[0,1] \rightarrow S[0,1]$.

To prove that $A D[0,1]$ is the largest $*$-subalgebra of $S[0,1]$, which admits unique extension of $\partial: D[0,1] \rightarrow S[0,1]$ we recall the following notions. Let, as before, $\mathcal{A}[x]$ denote the *-algebra of all polynomials with coefficients from a subalgebra $\mathcal{A} \subset S[0,1]$. An element $a \in S[0,1]$ is said to be

algebraic with respect to $\mathcal{A}$, if there exists a non-zero polynomial $p \in \mathcal{A}[x]$, such that $p(a)=0$;

transcendental with respect to $\mathcal{A}$, if $a$ is not algebraic over $\mathcal{A}$;

weakly transcendental with respect to $\mathcal{A}$, if $a \neq 0$, and for any non-zero idempotent $e \leq s(a)$ the element $e a$ is not integral with respect to $\mathcal{A}$.

Theorem 4.14. The *-algebra $A D[0,1]$ is the largest subalgebra of $S[0,1]$, containing the algebra $D[0,1]$, which admits unique extension of the derivation $\partial: D[0,1] \rightarrow S[0,1]$.

Proof. Suppose that $\mathcal{A}$ is a subalgebra of $S[0,1]$, such that $D[0,1] \subset A D[0,1] \subset \mathcal{A}$ and there exists a unique extension $\delta: \mathcal{A} \rightarrow S[0,1]$ of the derivation $\partial: D[0,1] \rightarrow S[0,1]$. We claim that $\mathcal{A} \subset A D[0,1]$.

Assume the contrary. Then there exists an element $a \in \mathcal{A}$ such that $a \notin A D[0,1]$. Let $\nabla$ be Boolean algebra of all idempotents in $S[0,1]$. The subset

$$
\nabla_{a}=\{e \in \nabla: e a \in A D[0,1]\}
$$

is non empty, since $0 \in \nabla_{a}$. Let

$$
e_{a}=\sup _{e \in \nabla_{a}} e .
$$

Take any elements $e, g \in \nabla_{a}$. Since $(e \vee g) a=e a+(g-e \wedge g) a \in A D[0,1]$, it follows that $e \vee g \in \nabla_{a}$, in other words the set $\nabla_{a}$ is closed under the operation $\vee$. Therefore there exists an increasing net $\left\{e_{i}\right\} \subset \nabla_{a}$ such that $e_{i} \uparrow e_{a}$. Thus $e_{a} \in \nabla_{a}$, because $e_{i} a \stackrel{\rho}{\longrightarrow} e_{a} a$ and $A D[0,1]$ is $\rho$-closed (see Proposition 4.8).

By the assumption, we have $a \notin A D[0,1]$, and therefore $e_{a} \neq 1$. By a construction, $e_{a}$ is the greatest element of $\nabla_{a}$, hence $f a \notin A D[0,1]$ for all $0 \neq f \leq \mathbf{1}-e_{a}$. It follows that $a\left(\mathbf{1}-e_{a}\right)$ is a weakly transcendental element with respect to $A D[0,1]$. Otherwise, there is an idempotent $0 \neq f \leq \mathbf{1}-e_{a}$ such that $f a$ is integral with respect to $A D[0,1]$. But $A D[0,1]$ is integrally closed (see Proposition 4.10), and hence we should have $f a \in A D[0,1]$, which is impossible due to the maximality of the element $e_{a}$.

Let $\mathcal{B}$ be a $*$-subalgebra generated by $\mathcal{A}$ and $A D[0,1]$. Since $a\left(\mathbf{1}-e_{a}\right)$ is a weakly transcendental element with respect to $A D[0,1],[5$, Proposition 3.7] implies that on $\mathcal{B}$ there exist derivations $\delta_{1}$ and $\delta_{2}$, extending $\partial_{A D}$ such that $\delta_{1}\left(a\left(1-e_{a}\right)\right)=0$ and $\delta_{2}\left(a\left(\mathbf{1}-e_{a}\right)\right)=\mathbf{1}-e_{a}$. Hence $\delta_{1}(a)=\partial_{A D}\left(a e_{a}\right)$ and $\delta_{2}(a)=\partial_{A D}\left(a e_{a}\right)+\mathbf{1}-e_{a}$. Thus the 
restrictions of $\delta_{1}$ and $\delta_{2}$ onto $\mathcal{A}$ are different extensions of $\partial$ onto $\mathcal{A}$, which contradicts the assumption that $\partial$ admits a unique extension onto $\mathcal{A}$. This completes the proof.

Remark 4.15. It should be pointed out that there are various extensions and generalizations of the classical derivation $\frac{d}{d t}$ as well as various classes of differentiable functions corresponding to such generalizations (see e.g. [42]). The special interest attached to the notion of approximate differentiation and its corresponding class $A D[0,1]$ is justified by the fact that the algebra $A D[0,1]$ is the largest subalgebra of $S[0,1]$ admitting a unique extension of the classical derivation $\frac{d}{d t}$.

\section{The ALGEBRA OF APPROXIMATELY DIFFERENTIABlE OPERATORS AFFILIATED WITH HYPERFINITE TYPE $\mathrm{II}_{1}$ FACTOR AND ITS DERIVATIONS}

In this section we introduce an analogue of approximately differentiable functions for hyperfinite type $\mathrm{II}_{1}$ factor $\mathcal{R}$. As in the commutative case, they form a regular *-subalgebra $A D(\mathcal{R})$ in the algebra $S(\mathcal{R})$. We show that there exists a derivation $\delta_{A D}$ : $A D(\mathcal{R}) \rightarrow S(\mathcal{R})$ which extends the classical approximate derivative $\partial_{A D}$, discussed in Section 4 .

The contents of this section complement and extend results from seminal work due to von Neumann [37]. In that work, a regular ring $C_{\infty}$ of continuous geometry for $\mathbb{C}$ was constructed starting with a sum of an increasing sequence of matrix rings over the field of complex numbers and then completed in the rank-metric. Our noncommutative analogue of approximately differentiable functions for hyperfinite type $\mathrm{II}_{1}$ factor $\mathcal{R}$, is defined as a completion in the rank-metric of a sum of an increasing sequence of matrix rings over $A D[0,1]$.

5.1. Hyperfinite $I I_{1}$-factor as an infinite tensor product. The idea of approximately differentiable operators for the hyperfinite type $\mathrm{II}_{1}$ factor $\mathcal{R}$ is based on the identification of $\mathcal{R}$ with the relative infinite tensor product of matrix algebras. We start with recalling this identification and refer the reader for further details concerning this construction to [19, 21, 43].

Let $M_{2}(\mathbb{C})$ be the algebra of all $2 \times 2$ matrices over the filed $\mathbb{C}$ of all complex numbers. We denote by $t r_{2}$ the normalised trace $t r_{2}$ on $M_{2}(\mathbb{C})$, that is $\operatorname{tr}_{2}\left(1_{2}\right)=1$, where $1_{2}$ is the $2 \times 2$ identity matrix.

The hyperfinite $I I_{1}$-factor $\mathcal{R}$ can be identified with the relative infinite tensor product

$$
(\mathcal{R}, \tau) \cong \bigotimes_{k=1}^{\infty}\left(M_{2}(\mathbb{C}), t r_{2}\right)
$$

such that $\tau$ is a faithful normal tracial state on $\mathcal{R}$. The construction of relative infinite tensor products is detailed in [50, Definition XIV.1.6].

For each $n \geq 1$, we may consider finite truncations of this infinite tensor product. Set $\mathcal{R}_{0}=\mathbb{C}$, and let

$$
\left(\mathcal{R}_{n}, \tau_{n}\right)=\bigotimes_{k=1}^{n}\left(M_{2}(\mathbb{C}), t r_{2}\right)=M_{2}(\mathbb{C})^{\otimes n}
$$

be the matrix space of $2^{n} \times 2^{n}$ matrices, with the normalised trace $\tau_{n}$.

There is a natural inclusion $\iota_{n}: \mathcal{R}_{n} \hookrightarrow \mathcal{R}$, for every $n \geq 0$, given by

$$
\iota_{n}(x)=x \otimes\left(\bigotimes_{k=n+1}^{\infty} 1_{m(k)}\right) \text {. }
$$


We will identify each $\mathcal{R}_{n}$ with its image $\iota_{n}\left(\mathcal{R}_{n}\right) \subset \mathcal{R}$. Each $\mathcal{R}_{n}$ is trivially a von Neumann subalgebra of $\mathcal{R}$, and the restriction of $\tau$ to $\mathcal{R}_{n}$ gives the trace $\tau_{n}$.

Thus, the spaces $\left(\mathcal{R}_{n}\right)_{n=0}^{\infty}$ form an increasing filtration of $\mathcal{R}$, and by definition of the infinite tensor product, the union $\bigcup_{n=0}^{\infty} \mathcal{R}_{n}$ is weak-* dense in $\mathcal{R}$.

Alternatively, it will also be useful to consider the spaces $\mathcal{R}_{n}$ as vector valued matrix spaces. In particular, for each $n \geq 1$, the space $\mathcal{R}_{n}$ is isomorphic to $M_{2}\left(\mathcal{R}_{n-1}\right)$, the space of $\mathcal{R}_{n-1}$-valued $2 \times 2$ matrices. This follows as $\mathcal{R}_{n}=\mathcal{R}_{n-1} \otimes M_{2}(\mathbb{C})$, by definition.

5.2. *-algebra of approximately differentiable operators. Let $D_{2}(\mathbb{C})$ be the diagonal subalgebra in $M_{2}(\mathbb{C})$ and consider the maximal abelian subalgebra $\mathcal{D}$ in $\mathcal{R}$, defined by

$$
\mathcal{D}=\bigotimes_{k=1}^{\infty}\left(D_{2}(\mathbb{C}), t r_{2}\right)
$$

It is known [51, Theorem 3.2] that $\mathcal{D}$ is a Cartan subalgebra in $\mathcal{R}$.

We identify

$$
(\mathcal{D}, \tau)=\bigotimes_{k=1}^{\infty} L_{\infty}(\{0,1\}, \nu)
$$

where $\nu(0)=\nu(1)=\frac{1}{2}$. The latter algebra is identified with $L_{\infty}(0,1)$ equipped with the usual Lebesgue integration.

We specify the $*$-isomorphism $\pi$ of the algebras $L_{\infty}[0,1]$ and $\mathcal{D}$.

Consider subsets $X_{k}=\bigcup_{l=0}^{2^{k-1}-1}\left[\frac{2 l}{2^{k}}, \frac{2 l+1}{2^{k}}\right), k \in \mathbb{N}$. Define the mapping

$$
\pi\left(\chi_{X_{k}}\right)=\left(\bigotimes_{i=1}^{k-1} 1_{i}\right) \otimes\left(\begin{array}{ll}
1 & 0 \\
0 & 0
\end{array}\right) \otimes\left(\bigotimes_{i=k+1}^{\infty} 1_{i}\right)
$$

where $1_{i}$ is the $2 \times 2$ identity matrix. The system $\left\{2 \chi_{x_{k}}-1: k \in \mathbb{N}\right\}$ is the Rademacher system of functions on $[0,1]$, that is a system of independent random variables taking values 1 and -1 with probability $1 / 2$. The span of $\left\{1, \chi_{X_{k}}: k \in \mathbb{N}\right\}$ is dense in $L_{\infty}[0,1]$ in measure. Therefore, the mapping $\pi$ uniquely extends to a $*$-isomorphism $\pi: L_{\infty}[0,1] \longrightarrow \mathcal{D}$. Since the $*$-isomorphism $\pi$ preserves the trace, it follows from Proposition 2.1 that it extends up to $*$-isomorphism $\pi: S[0,1] \rightarrow S(\mathcal{D})$.

Therefore, throughout this section we do not distinguish between the $*$-algebra $\mathcal{D}$ (respectively, $S(\mathcal{D})$ ) and the $*$-algebra $L_{\infty}[0,1]$ (respectively, $S[0,1]$ ). In particular, we identify $S(0,1)$ with a $*$-subalgebra of $S(\mathcal{R})$.

For $n \geq 1$, the algebra $\mathcal{R}_{n}=M_{2}(\mathbb{C})^{\otimes n}$ is spanned by the "matrix units" $e_{\mathbf{i}, \mathbf{j}}$. Here, $\mathbf{i}=\left(i_{k}\right)_{k=0}^{n-1} \in\{0,1\}^{n}, \mathbf{j}=\left(j_{k}\right)_{k=0}^{n-1} \in\{0,1\}^{n}$, and

$$
e_{\mathbf{i}, \mathbf{j}}=\bigotimes_{k=0}^{n-1} e_{i_{k}, j_{k}}
$$

Therefore, each matrix $x \in \mathcal{R}_{n}$ has the form

$$
x=\sum_{\mathbf{i}, \mathbf{j}} a_{\mathbf{i}, \mathbf{j}} e_{\mathbf{i}, \mathbf{j}}, \quad a_{\mathbf{i}, \mathbf{j}} \in \mathbb{C} .
$$

For each pair induces $\mathbf{i}=\left(i_{k}\right)_{k=0}^{n-1} \in\{0,1\}^{n}, \mathbf{j}=\left(j_{k}\right)_{k=0}^{n-1} \in\{0,1\}^{n}$ consider a mapping from $S(\mathcal{D}) e_{\mathbf{i}, \mathbf{i}}$ onto $S(\mathcal{D}) e_{\mathbf{j}, \mathbf{j}}$ defined as follows

$$
\pi(x) e_{\mathbf{i}, \mathbf{i}} \hookrightarrow e_{\mathbf{j}, \mathbf{i}} \pi(x) e_{\mathbf{i}, \mathbf{j}}, \pi(x) \in S(\mathcal{D}) .
$$


It induces a mapping from $S[0,1] \chi_{\left[\frac{i}{2^{n}}, \frac{i+1}{2^{n}}\right]}$ onto $S[0,1] \chi_{\left[\frac{j}{2^{n}}, \frac{j+1}{2^{n}}\right]}$ defined as

$$
x(t) \chi_{\left[\frac{i}{2^{n}}, \frac{i+1}{2^{n}}\right]} \longrightarrow x\left(t+\frac{j-i}{2^{n}}\right) \chi_{\left[\frac{j}{2^{n}}, \frac{j+1}{2^{n}}\right]},
$$

where $i=\sum_{k=0}^{n-1} i_{k} 2^{n-k-1}, j=\sum_{k=0}^{n-1} j_{k} 2^{n-k-1}$.

Let $A D(\mathcal{D})$ be the (commutative) *-algebra of all approximately differentiable functions on $(0,1)$ (see Section 4), which we identify with a subspace of $S(\mathcal{R})$.

Recall that the complete metric $\rho$ on $S(\mathcal{R})$ is defined by setting

$$
\rho(x, y)=\tau(l(x-y))=\tau(r(x-y)), \quad x, y \in S(\mathcal{R}) .
$$

As we mentioned above in Section 2.3 the rank-metric $\rho$ was firstly introduced by J. von Neumann in [36] (see also [39, pp. 160-161]).

We now introduce a noncommutative analogue of the algebra $A D[0,1]$, discussed at length in Section 4 ,

Definition 5.1. Let $n \geq 0$ and let $\mathcal{A}_{n}:=A D\left(\mathcal{R}_{n}\right)$ be the $*$-subalgebra of $S(\mathcal{R})$ generated by $\mathcal{R}_{n}$ and $A D(\mathcal{D})$. We define the *-algebra $A D(\mathcal{R})$ of approximately differentiable operators in $S(\mathcal{R})$ by setting

$$
A D(\mathcal{R})=\bar{\bigcup}_{n \geq 0} \mathcal{A}_{n}{ }^{\rho} .
$$

It is important to emphasize the connection of Definition 5.1 with seminal von Neumann paper [37]. Indeed, the algebra $A D(\mathcal{R})$ contains a regular ring of continuous geometry for $\mathbb{C}$, introduced in [37]. Recall that

$$
\mathcal{R}_{\infty}={\overline{\bigcup_{n \geq 0} \mathcal{R}_{n}}}^{\rho}
$$

is a continuous geometry for $\mathbb{C}$ (see [37, Theorems $\mathrm{D}$ and $\mathrm{E}]$ ), and contained in $A D(\mathcal{R})$. Below, in Proposition 5.3 we shall prove that the algebra $A D(\mathcal{R})$ is a proper *subalgebra in $S(\mathcal{R})$ and this may be seen as an extension of von Neumann results [37, Theorem E].

To establish the regularity of the algebra $A D(\mathcal{R})$ and to introduce a derivation on $A D(\mathcal{R})$, we prove firstly the following auxiliary result for the *-algebra $A D\left(\mathcal{R}_{n}\right)$.

Lemma 5.2. The *-algebra $A D\left(\mathcal{R}_{n}\right)$ is regular and every $x \in A D\left(\mathcal{R}_{n}\right)$ can be written, not necessarily uniquely, as

$$
x=\sum_{U \in \Pi_{n}} x_{U} U, \quad x_{U} \in A D(\mathcal{D})
$$

Here $\Pi_{n}$ is the collection of all permutation matrices from $\mathcal{R}_{n}$.

Proof. Note that the algebra $A D\left(\mathcal{R}_{n}\right)$ is generated by $A D(\mathcal{D})$ and $\Pi_{n}$. That is, any $x \in A D\left(\mathcal{R}_{n}\right)$ can be written as a linear span of monomials of the form

$$
a_{1} U_{1} a_{2} U_{2} \cdots a_{m} U_{m}
$$

for some $m \in \mathbb{N}$ and $a_{k} \in A D(\mathcal{D})$ and $U_{k} \in \Pi_{n}$ for every $1 \leq k \leq m$. Note that

$$
U a U^{-1} \in A D(\mathcal{D})
$$


for every $a \in A D(\mathcal{D})$ and for every permutation matrix $U$. Since $\prod_{l=1}^{k-1} U_{l}$ is again a permutation matrix, it follows that there exists $b_{k} \in A D(\mathcal{D})$ such that

$$
\prod_{l=1}^{k-1} U_{l} \cdot a_{k}=b_{k} \cdot \prod_{l=1}^{k-1} U_{l}
$$

In particular, $A D\left(\mathcal{R}_{n}\right)$ is a matrix ring over $A D(\mathcal{D})$. By Proposition 4.8 the algebra $A D(\mathcal{D})=A D[0,1]$ is regular. Since every matrix ring over a regular ring is also regular (see e.g. [46, Theorem 3]), it follows that $A D\left(\mathcal{R}_{n}\right)$ is regular.

By repeated application of (5.3) , we have that

$$
a_{1} U_{1} a_{2} U_{2} \cdots a_{m} U_{m}=a_{1} b_{2} \cdots b_{m} \cdot U_{1} \cdots U_{m} .
$$

Hence, any $x \in A D\left(\mathcal{R}_{n}\right)$ can be represented as in (5.2).

Proposition 5.3. The subalgebra $A D(\mathcal{R})$ is a proper regular *-subalgebra in $S(\mathcal{R})$ which is dense in $S(\mathcal{R})$ in the measure topology.

Proof. By Lemma 5.2 the algebra $A D\left(\mathcal{R}_{n}\right)$ is a regular $*$-algebra. Since $\left\{A D\left(\mathcal{R}_{n}\right)\right\}_{n=0}^{\infty}$ is an increasing sequence of subalgebras in $S(\mathcal{R})$, it follows that $\mathcal{A}:=\bigcup_{n=0}^{\infty} A D\left(\mathcal{R}_{n}\right)$ is also a regular subalgebra in $S(\mathcal{R})$. Hence, $A D(\mathcal{R})$ is also regular as the closure of a regular algebra with respect to the metric $\rho$ (see e.g. [25, Theorem 19.6]).

Let $x \in \mathcal{R}$ and $\|x\| \leq 1$. Since the $*$-subalgebra $\bigcup_{n=1}^{\infty} \mathcal{R}_{n} \subset A D(\mathcal{R})$ is dense in $\mathcal{R}$ in the strong operator topology, there exists a net $\left\{x_{\alpha}\right\}$ from the unit ball in $\bigcup_{n=1}^{\infty} \mathcal{M}_{n}$ such that $x_{\alpha} \stackrel{\text { so }}{\longrightarrow} x$. Then $\tau\left(\left(x_{\alpha}-x\right)^{*}\left(x_{\alpha}-x\right)\right) \rightarrow 0$ (see [47, Page 130]). This means that the net $\left\{x_{\alpha}\right\}$ converges to $x$ in the norm $\|\cdot\|_{2}$, where $\|z\|_{2}=\sqrt{\tau\left(z^{*} z\right)}, z \in \mathcal{R}$. Since convergence in the norm $\|\cdot\|_{2}$ implies convergence in measure topology (see [35, Theorem 5]), the net $\left\{x_{\alpha}\right\}$ converges to $x$ in the measure topology. So, $\bigcup_{n=1}^{\infty} \mathcal{R}_{n}$ is dense in the measure topology in $\mathcal{R}$, and is, therefore, dense in $S(\mathcal{R})$.

Now we show that $A D(\mathcal{R})$ is a proper subalgebra of $S(\mathcal{R})$. For every $n \in \mathbb{N}$ take a continuous piecewise-linear function $h_{n}$ on $[0,1]$ defined as follows

$$
h_{n}(t)= \begin{cases}0, & \text { if } t=\frac{2 l}{2^{16^{n}}}, l=0,1,2, \ldots, 2^{16^{n}-1} \\ \frac{1}{2^{16^{n}-4^{n+1}},}, & \text { if } t=\frac{2 l+1}{2^{16^{n}}}, l=0,1,2, \ldots, 2^{16^{n}-1}-1 \\ \text { linear, } & \text { if } \frac{l}{2^{16^{n}}} \leq t \leq \frac{l+1}{2^{16^{n}}}, l=0,1,2, \ldots, 2^{16^{n}}-1 .\end{cases}
$$

This function coincides with that defined in 28] for the sequences $\left\{k_{n}=2^{4^{n+1}}\right\}$ and $\left\{d_{n}=2^{-16^{n}}\right\}$ (see [28, p. 6]). Note that $h_{n}(\cdot)$ is differentiable on all of points $[0,1]$ excepting the finite number of points $t=\frac{l}{2^{16^{n}}}, l=0,1,2, \ldots, 2^{16^{n}}$. Setting

$$
h(t)=\sum_{k=0}^{\infty} h_{k}(t), \quad t \in[0,1]
$$

where the series is, in fact, uniformly convergent due to [28, p. 7]. In particular, the function $h$ is continuous but nowhere approximately differentiable (see [28, Theorem $1])$.

Let $n \in \mathbb{N}$ and $t \in\left[0,2^{-16^{n}+1}\right]$. For every $l \in\left\{0, \ldots, 2^{16^{n}-1}-1\right\}$, by the definition of $h_{k}(t)$ it follows that

$$
h_{k}\left(t+\frac{2 l}{2^{16^{n}}}\right)=h_{k}(t), \quad t \in[0,1]
$$


for $k \geq n$, and therefore the difference

$$
h\left(\cdot+\frac{2 l}{2^{16^{n}}}\right)-\left.h(\cdot)\right|_{\left[0,2^{-16^{n}+1}\right]}
$$

is a finite sum of the almost everywhere approximately differentiable functions on $\left[0,2^{-16^{n}+1}\right]$. So,

$$
h\left(\cdot+\frac{2 l}{2^{16^{n}}}\right)-\left.h(\cdot)\right|_{\left[0,2^{-16^{n}+1}\right]} \in A D\left[0,2^{-16^{n}+1}\right]
$$

for all $n \in \mathbb{N}$ and for all $l \in\left\{0, \ldots, 2^{16^{n}-1}-1\right\}$.

Set $\bar{h}=\pi(h)$, where $\pi: L_{\infty}[0,1] \longrightarrow \mathcal{D}$ is a $*$-isomorphism defined prior to the statement of the proposition.

Let us show that $\rho(\bar{h}, a)=1$ for all $a \in A D\left(\mathcal{R}_{m}\right)$, where $m=16^{n}-1, n \geq 1$. Fix $a \in A D\left(\mathcal{R}_{m}\right)$.

Consider a system of matrix units $\left\{e_{\mathbf{i}, \mathbf{j}}\right\}_{\mathbf{i} \in \mathbf{I}}$ in $\mathcal{R}_{m}$, here $\mathbf{I}=\left\{\mathbf{i}: \mathbf{i}=\left(i_{k}\right)_{k=0}^{m-1} \in\{0,1\}^{m}\right\}$. Let $\mathcal{B}_{m}$ be a $*$-algebra in $S(\mathcal{R})$ generated by $S(\mathcal{D})$ and $\mathcal{R}_{m}$. We shall identify $\mathcal{B}_{m}$ with the matrix $*$-algebra $M_{2^{m}}\left(S(\mathcal{D}) e_{\mathbf{0}, \mathbf{0}}\right)$ via $*$-isomorphism

$$
\Psi_{m}: x \rightarrow\left(e_{\mathbf{0}, \mathbf{i}} x e_{\mathbf{j}, \mathbf{0}}\right)_{\mathbf{i}, \mathbf{j} \in \mathbf{I}},
$$

where $\mathbf{0}=(0, \ldots, 0)$. Observe that $\Psi_{m}(a) \in M_{2^{m}}\left(A D(\mathcal{D}) e_{\mathbf{0}, \mathbf{0}}\right)$.

Note that the $*$-isomorphism $\pi$ sends a function $h\left(\cdot+\frac{2 l}{2^{16^{n}}}\right) \chi_{\left[0, \frac{2 l}{2^{16^{n}}}\right)}(\cdot) \in S[0,1]$ to the element of the form $e_{\mathbf{0}, \mathbf{i}} \bar{h} e_{\mathbf{i}, \mathbf{0}} \in S(\mathcal{D}) e_{\mathbf{0}, \mathbf{0}}$, where $2 l=\sum_{k=0}^{m-1} i_{k} 2^{m-k-1}$ for $\mathbf{i}=\left(i_{k}\right)_{k=0}^{m-1} \in$ $\{0,1\}^{m}$. Combining this observation with (5.4), we arrive at

$$
e_{\mathbf{0}, \mathbf{i}} \bar{h} e_{\mathbf{i}, \mathbf{0}}=\bar{h} e_{\mathbf{0}, \mathbf{0}}+r_{\mathbf{i}}
$$

where $r_{\mathbf{i}} \in A D(\mathcal{D}) e_{\mathbf{0}, \mathbf{0}}$ for all $\mathbf{i} \in \mathbf{I}$. Recalling that $h$ is nowhere approximately differentiable and that the element $\Psi_{m}(a)$ belongs $M_{2^{m}}\left(A D(\mathcal{D}) e_{\mathbf{0 , 0}}\right)$, and appealing to Proposition 4.12, we infer that the matrix $\Psi_{m}(a-\bar{h}-r)$ is invertible in $M_{2^{m}}\left(S(\mathcal{D}) e_{\mathbf{0}, \mathbf{0}}\right)$, where $r=\sum_{\mathbf{i} \in \mathbf{I}} r_{\mathbf{i}} e_{\mathbf{i}, \mathbf{i}}$. In other words, the support projection of the element $a-\bar{h}-r$ is the identity of the algebra $\mathcal{R}$. Hence, $\rho(a-r, \bar{h})=1$. Observing that the definition of the element $r \in A D(\mathcal{D})$, does not depend on the choice of $a$, we may replace $a$ with $a+r$ and obtain that $\rho(a, \bar{h})=1$. Thus $\rho(a, \bar{h})=1$ for all $a \in \bigcup_{m \geq 0} A D\left(\mathcal{R}_{m}\right)$, hence $\bar{h} \in S(\mathcal{R}) \backslash A D(\mathcal{R})$. The proof is completed.

5.3. Approximate derivation on the algebra $A D(\mathcal{R})$. We now construct the derivation $\delta_{A D}: A D(\mathcal{R}) \rightarrow S(\mathcal{R})$, which extends the approximate derivative $\partial_{A D}$ on the $*$-algebra $A D(\mathcal{D})=A D[0,1]$, introduced in Section 4 . We start by constructing a tower of derivations on the $*$-algebras $A D\left(\mathcal{R}_{n}\right), n \geq 1$.

Recall that $\Pi_{n}$ is the collection of all permutation matrices from $\mathcal{R}_{n}$. By Lemma 5.2 every element $x \in A D\left(\mathcal{R}_{n}\right)$ can be represented as $x=\sum_{U \in \Pi_{n}} x_{U} U$ for some $x_{U} \in$ $A D(\mathcal{D})$.

We define

$$
\delta_{n}(x)=\sum_{U \in \Pi_{n}} \partial_{A D}\left(x_{U}\right) U, \quad n \geq 1
$$

For convenience, we also denote $\delta_{0}=\partial_{A D}$.

Lemma 5.4. The mapping $\delta_{n}: A D\left(\mathcal{R}_{n}\right) \rightarrow S(\mathcal{R}), n \geq 1$, given by (5.5), is a welldefined linear mapping. 
Proof. Let $n \geq 1$ be fixed. It is sufficient to show that if $\sum_{k=1}^{m} x_{k} A_{k}=0$ for some $x_{k} \in A D(\mathcal{D})$ and $A_{k} \in \mathcal{R}_{n}, k=1, \ldots, m, m \in \mathbb{N}$, then $\sum_{k=1}^{m} \partial_{A D}\left(x_{k}\right) A_{k}=0$.

Recall that for $n \geq 1$, the algebra $\mathcal{R}_{n}=M_{2}(\mathbb{C})^{\otimes n}$ is spanned by the "matrix units" $e_{\mathbf{i}, \mathbf{j}}$, where, $\mathbf{i}=\left(i_{k}\right)_{k=0}^{n-1} \in\{0,1\}^{n}, \mathbf{j}=\left(j_{k}\right)_{k=0}^{n-1} \in\{0,1\}^{n}$, and $e_{\mathbf{i}, \mathbf{j}}=\bigotimes_{k=0}^{n-1} e_{i_{k}, j_{k}}$. Therefore, each matrix $A_{k} \in \mathcal{R}_{n}$ has the form.

$$
A_{k}=\sum_{\mathbf{i}, \mathbf{j}} a_{\mathbf{i}, \mathbf{j}}^{k} e_{\mathbf{i}, \mathbf{j}}, \quad a_{\mathbf{i}, \mathbf{j}}^{k} \in \mathbb{C} .
$$

For each pair of fixed indices $\mathbf{i}_{\mathbf{0}}, \mathbf{j}_{\mathbf{0}}$ multiplying the equality $\sum_{k=1}^{m} x_{k} A_{k}=0$ by $e_{\mathbf{i}_{\mathbf{0}}, \mathbf{i}_{\mathbf{0}}}$ on the left side and by $e_{\mathbf{j}_{\mathbf{o}}, \mathbf{i}_{\mathbf{0}}}$ on the right, we obtain that

$$
\sum_{k=1}^{m} a_{\mathbf{i}_{0}, \mathbf{j}_{\mathbf{0}}}^{k} x_{k} e_{\mathbf{i}_{\mathbf{0}}, \mathbf{i}_{\mathbf{0}}}=0
$$

Note that $e_{\mathbf{i}_{0}, \mathbf{i}_{\mathbf{0}}} \in A D(\mathcal{D})$, and $e_{\mathbf{i}_{0}, \mathbf{i}_{0}}$ is a projection. Therefore, since the derivation $\partial_{A D}$ vanishes on projections, it follows that from the Leibniz rule that

$$
\sum_{k=1}^{m} a_{\mathbf{i}_{\mathbf{0}}, \mathbf{j}_{\mathbf{0}}}^{k} \partial_{A D}\left(x_{k}\right) e_{\mathbf{i}_{\mathbf{0}}, \mathbf{i}_{\mathbf{0}}}=\partial_{A D}\left(\sum_{k=1}^{m} a_{\mathbf{i}_{\mathbf{0}}, \mathbf{j}_{\mathbf{0}}}^{k} x_{k} e_{\mathbf{i}_{\mathbf{0}}, \mathbf{i}_{\mathbf{0}}}\right)=0 .
$$

Multiplying the last equality by $e_{\mathbf{i}_{\mathbf{0}} \mathbf{j} \mathbf{0}}$ from the right, we obtain

$$
\sum_{k=1}^{m} a_{\mathbf{i}_{\mathbf{o}}, \mathbf{j}_{\mathbf{0}}}^{k} \partial_{A D}\left(x_{k}\right) e_{\mathbf{i}_{\mathbf{o}}, \mathbf{j}_{\mathbf{0}}}=0 \text {. }
$$

Hence,

$$
\sum_{k=1}^{m} \partial_{A D}\left(x_{k}\right) A_{k}=\sum_{k=1}^{m} \sum_{\mathbf{i}, \mathbf{j}} a_{\mathbf{i}, \mathbf{j}}^{k} \partial_{A D}\left(x_{k}\right) e_{\mathbf{i}, \mathbf{j}}=\sum_{\mathbf{i}, \mathbf{j}} \sum_{k=1}^{m} a_{\mathbf{i}, \mathbf{j}}^{k} \partial_{A D}\left(x_{k}\right) e_{\mathbf{i}, \mathbf{j}}=0,
$$

which completes the proof.

Recall that we identify the corresponding elements from $S(\mathcal{D})$ and from $S(0,1)$.

Proposition 5.5. Let $n \geq 1$ and let $U \in \Pi_{n}$ be a permutation matrix. Then there exists a permutation $\gamma:[0,1] \rightarrow[0,1]$ of dyadic intervals, such that

$$
U c U^{-1}=c \circ \gamma, \quad \forall c \in S[0,1] .
$$

Moreover, if, in addition, $c \in A D[0,1]$, then $U c U^{-1} \in A D[0,1]$ and $\partial_{A D}(c \circ \gamma)=$ $\partial_{A D}(c) \circ \gamma$.

Proof. Let $\mathbf{I}=\left\{\mathbf{i}: \mathbf{i}=\left(i_{k}\right)_{k=0}^{n-1} \in\{0,1\}^{n}\right\}$. For $\mathbf{i} \in \mathbf{I}$ set

$$
X_{\mathbf{i}}=\bigcap_{s=0}^{n-1} Y_{s+1}
$$

where $Y_{s+1}=X_{s+1}$ for $i_{s}=0$ and $Y_{s+1}=[0,1) \backslash X_{s+1}$ for $i_{s}=1$. Here $\left\{X_{k}: k \in \mathbb{N}\right\}$ is the system of subsets in $[0,1]$ defined before (5.1). Then $\left\{X_{\mathbf{i}}: \mathbf{i} \in \mathbf{I}\right\}$ is a partition of $[0,1]$ into dyadic intervals of the lengths $1 / 2^{n}$. Using (5.1) we obtain that

$$
\pi\left(\chi_{X_{\mathbf{i}}}\right)=\prod_{s=0}^{n-1} \pi\left(\chi_{Y_{s+1}}\right)=\bigotimes_{s=0}^{n-1} e_{i_{s}, i_{s}}=e_{\mathbf{i}, \mathbf{i}}
$$

for all $\mathbf{i} \in \mathbf{I}$. 
Since any permutation matrix $U \in \Pi_{n}$ induces a permutation of the system $\left\{e_{\mathbf{i}, \mathbf{i}}: \mathbf{i} \in\right.$ I\}, we have that $U e_{\mathbf{i}, \mathbf{i}} U^{-1}=e_{\sigma(\mathbf{i}), \sigma(\mathbf{i})}$, where $\sigma$ is a permutation of $\mathbf{I}$. Then (5.6) gives us $U \pi\left(\chi_{\mathbf{i}}\right) U^{-1}=\pi\left(\chi_{\sigma(\mathbf{i})}\right)$. Thus $\sigma$ induces a mapping $\gamma:[0,1] \rightarrow[0,1]$ which acts as a permutation of dyadic intervals and $U \pi\left(\chi_{X_{\mathbf{i}}}\right) U^{-1}=\pi\left(\chi_{X_{\mathbf{i}}}\right) \circ \gamma$ for all $\mathbf{i} \in \mathbf{I}$. Further for $k>n$, the subset $X_{k}$ the collection of disjoint dyadic intervals with the lengths $1 / 2^{k}$, and therefore $\gamma$ permutes the elements of $X_{k}$. Hence, $U \pi\left(\chi_{X_{k}}\right) U^{-1}=\pi\left(\chi_{X_{k}}\right)=\pi\left(\chi_{X_{k}}\right) \circ \gamma$ for all $k>n$. Since a linear span of the system $\left\{1, \chi_{X_{\mathbf{i}}}: \mathbf{i} \in \mathbf{I}\right\} \cup\left\{\chi_{X_{k}}: k>n\right\}$ is dense in the measure topology in $S[0,1]$, it follows that $U c U^{-1}=c \circ \gamma$ for all $c \in S[0,1]$.

Let $c \in A D[0,1]$. In order to prove that $U c U^{-1} \in A D[0,1]$ it suffices to show $c \circ \gamma \in$ $A D[0,1]$, where $\gamma(t)=(t+r)(\bmod 1), r \in(0,1)$ is a dyadic rational. By Proposition 4.7 it suffices to consider the case $c \in D[0,1]$. Let $c$ has a finite derivative at each point of a subset $A \subset[0,1]$ with complete measure. Then a subset $\{A+r\}$ is also has complete measure, and therefore the intersection $A \cap\{A+r\}$ also has a complete measure. For every point $t$ of this intersection there exist finite derivatives $c^{\prime}(t)$ and $c^{\prime}(\{t+r\})$. This means that $c \circ \gamma \in D[0,1]$.

Finally, the equality $\partial_{A D}(c(\{t+r\}))=\left(\partial_{A D}(c)\right)(\{t+r\})$ implies that $\partial_{A D}(c \circ \gamma)=$ $\partial_{A D}(c) \circ \gamma$. The proof is complete.

Next, we show that the sequence $\left\{\delta_{n}\right\}_{n \geq 1}$, defined by (5.5) on the increasing sequence of algebras $A D\left(\mathcal{R}_{n}\right)$, is a sequence of derivations such that each of the subsequent derivation is an extension of the previous one and all of them vanish on $\cup_{n \geq 1} \mathcal{R}_{n}$.

Proposition 5.6. Let $\delta_{n}: A D\left(\mathcal{R}_{n}\right) \rightarrow S(\mathcal{R})$ be the mapping, defined by (5.5). For every $n \geq 0, \delta_{n}$ is a derivation and

$$
\left.\delta_{n+1}\right|_{A D\left(\mathcal{R}_{n}\right)}=\delta_{n},\left.\quad \delta_{n}\right|_{\mathcal{R}_{n}}=0 .
$$

In particular, $\left.\delta_{n}\right|_{A D(\mathcal{D})}=\partial_{A D}, n \geq 1$.

Proof. We show firstly that $\delta_{n}$ is a derivation for every $n \geq 1$. By the definition of $\delta_{n}$, it suffices to verify the Leibniz rule for $x=a U$ and $y=b V$ with $a, b \in A D(\mathcal{D})$ and $U, V \in \Pi_{n}$.

Let $U \in \Pi_{n}$ be fixed permutation matrix. By Proposition 5.5 there exists a suitable permutation $\gamma:[0,1] \rightarrow[0,1]$ of dyadic intervals, such that

$$
U c U^{-1}=c \circ \gamma, \quad \forall c \in S[0,1] .
$$

In particular,

$$
U c U^{-1} \in A D[0,1], \quad \forall c \in A D[0,1]
$$

Given that the permutation $\gamma$ commutes with the approximate derivative $\partial_{A D}$ we obtain that

$$
\partial_{A D}\left(U c U^{-1}\right)=\partial_{A D}(c \circ \gamma)=\partial_{A D}(c) \circ \gamma=U \partial_{A D}(c) U^{-1}, \quad \forall c \in A D[0,1] .
$$

Therefore, we have

$$
\delta_{n}(x y)=\delta_{n}\left(a \cdot U b U^{-1} \cdot U V\right)=\partial_{A D}\left(a \cdot U b U^{-1}\right) \cdot U V .
$$

Using the Leibniz rule for the elements $a, U b U^{-1} \in A D[0,1]$ and (5.7) we infer that $\partial_{A D}\left(a \cdot U b U^{-1}\right)=\partial_{A D}(a) \cdot U b U^{-1}+a \cdot \partial_{A D}\left(U b U^{-1}\right)=\partial_{A D}(a) \cdot U b U^{-1}+a U \cdot \partial_{A D}(b) U^{-1}$.

Hence,

$$
\begin{aligned}
\delta_{n}(x y) & =\left(\partial_{A D}(a) \cdot U b U^{-1}+a U \cdot \partial_{A D}(b) U^{-1}\right) U V= \\
& =\partial_{A D}(a) U \cdot b V+a U \cdot \partial_{A D}(b) V=\delta_{n}(x) y+x \delta_{n}(y) .
\end{aligned}
$$


Thus, the Leibniz rule is satisfied for $x, y$, and therefore, $\delta_{n}$ is a derivation on $A D\left(\mathcal{R}_{n}\right)$. Since for any $x \in \mathcal{R}_{n}$ in the representation $x=\sum_{U \in \Pi_{n}} x_{U} U$ every $x_{U}$ is a constant, it follows immediately from the definition of $\delta_{n}$, that $\left.\delta_{n}\right|_{\mathcal{R}_{n}}=0$. The equality $\left.\delta_{n}\right|_{A D(\mathcal{D})}=$ $\partial_{A D}$ also follows directly, because for $x \in A D(\mathcal{D})$ the representation of $x$ in the form $x=$ $\sum_{U \in \Pi_{n}} x_{U} U$ involves only the identical permutation matrix and so the required equality follows immediately from (5.5). It remains to show that $\left.\delta_{n+1}\right|_{A D\left(\mathcal{R}_{n}\right)}=\delta_{n}, \quad n \geq 0$.

Define a derivation $\delta_{n}^{\prime}: A D\left(\mathcal{R}_{n}\right) \rightarrow S(\mathcal{R})$ by setting

$$
\delta_{n}^{\prime}=\delta_{n}-\left.\delta_{n+1}\right|_{A D\left(\mathcal{R}_{n}\right)} .
$$

We have

$$
\left.\delta_{n}^{\prime}\right|_{A D(\mathcal{D})}=0,\left.\quad \delta_{n}^{\prime}\right|_{\mathcal{R}_{n}}=0 .
$$

Since $A D\left(\mathcal{R}_{n}\right)$ is generated by $A D(\mathcal{D})$ and $\mathcal{R}_{n}$ it follows from the Leibniz rule that the derivation $\delta_{n}^{\prime}$ vanishes on $A D\left(\mathcal{R}_{n}\right)$. This proves the claim.

We are now in a position to construct a noncommutative analogue of the approximate derivative $\partial_{A D}$.

Theorem 5.7. There exists a derivation $\delta_{A D}: A D(\mathcal{R}) \rightarrow S(\mathcal{R})$ such that $\left.\delta\right|_{A D(\mathcal{D})}=$ $\partial_{A D}$

Proof. Let $\delta_{n}: A D\left(\mathcal{R}_{n}\right) \rightarrow S(\mathcal{R}), n \geq 0$, be the derivation, given by (5.5).

Consider the $*$-subalgebra $\mathcal{A}=\bigcup_{n \geq 0} A D\left(\mathcal{R}_{n}\right)$. Define the mapping $\delta: \mathcal{A} \rightarrow S(\mathcal{R})$ by setting $\left.\delta\right|_{A D\left(\mathcal{R}_{n}\right)}=\delta_{n}$. By Proposition 5.6 we have $\left.\delta_{n+1}\right|_{A D\left(\mathcal{R}_{n}\right)}=\delta_{n}$, and therefore, $\delta$ is a well-defined mapping. It is clear that $\delta$ is a derivation.

By Lemma 2.4 the derivation $\delta$ is continuous with respect to the metric $\rho$. By definition, $A D(\mathcal{R})={\overline{\bigcup_{n \geq 0} A D\left(\mathcal{R}_{n}\right)}}^{\rho}$, and so $\delta$ extends up to a derivation $\delta_{A D}: A D(\mathcal{R}) \rightarrow$ $S(\mathcal{R})$. Since $\left.\delta_{n}\right|_{A D(\mathcal{D})}=\partial_{A D}$, it follows that $\left.\delta_{A D}\right|_{A D(\mathcal{D})}=\partial_{A D}$, which completes the proof.

Next, we show that the noncommutative approximate derivative $\delta_{A D}(\mathcal{R})$ is not spatial.

Proposition 5.8. Let $\delta_{A D}: A D(\mathcal{R}) \rightarrow S(\mathcal{R})$ be as in Theorem 5.7. There is no $a \in S(\mathcal{R})$ such that $\delta_{A D}(x)=[a, x]$ for all $x \in A D(\mathcal{R})$.

Proof. Suppose, by contradiction, that there exists an element $a \in S(\mathcal{R})$ such that $\delta_{A D}(x)=[a, x]$ for all $x \in \mathcal{B}$. Since $S(\mathcal{R})$ equipped with the measure topology is a topological $*$-algebra it follows that, in particular, $\delta_{A D}$ is continuous with respect to the measure topology. Since $\left.\delta_{A D}\right|_{A D(\mathcal{D})}=\partial_{A D}$, it follows that $\partial_{A D}$ is also continuous in the measure topology. Furthermore, the algebra $A D(\mathcal{D})=A D[0,1]$ contains all projections from $\mathcal{D}=L_{\infty}[0,1]$ (see Proposition 4.6) and is, therefore, contained in the closure in measure topology of the set of all linear combinations of all projections. Since $\partial_{A D}$ vanishes on projections and is continuous in the measure topology, it follows that $\partial_{A D}=0$, and therefore, $\delta_{n}=0$. Hence, $\delta=0$, which is a contradiction.

Remark 5.9. We note that $A D(\mathcal{R})$ contains a regular ring of continuous geometry for $\mathbb{C}$, namely, $\mathcal{R}_{\infty}={\overline{\bigcup_{n \geq 0} \mathcal{R}_{n}}}^{\rho}$ is a continuous geometry by [37, Theorems D and E]. Furthermore, the ring $\mathcal{R}_{\infty}$ is a proper subalgebra of $A D(\mathcal{R})$. Indeed, $\left.\delta_{A D}\right|_{\mathcal{R}_{\infty}}=0$, because by Proposition $\left.5.6 \delta_{A D}\right|_{\mathcal{R}_{n}}=0$ for all $n \geq 0$ and $\delta_{A D}$ is $\rho$-continuous by Proposition 2.4. On the other hand, $\delta_{A D}$ is non trivial. Thus $\mathcal{R}_{\infty}$ is a proper subalgebra of $A D(\mathcal{R})$. 


\section{An example of a Derivation on a CARTAN MASA Which does not extend TO $S(\mathcal{M})$}

In this section, we prove that there is a derivation on the algebra $\mathcal{D}$ with values in $S(\mathcal{D})$, which can not be extended up to a derivation on $S(\mathcal{R})$. Using Connes-FeldmannWeiss theorem we also prove analogous result for any Cartan masa in a hyperfinite type $\mathrm{II}_{1}$ factor $\mathcal{R}$.

Let, as before, $\mathcal{D}$ be the "diagonal" masa in $\mathcal{R}$ (see Section 5.2). As before, we identify $\mathcal{D}=L_{\infty}[0,1]$ and view $S(\mathcal{D})=S[0,1]$ as a $*$-subalgebra of $S(\mathcal{R})$.

Theorem 6.1. Let $\delta: A D(\mathcal{D}) \rightarrow S(\mathcal{D})$ be a derivation, such that $\left.\delta\right|_{A D[0,1 / 2]}=\partial_{A D}$ and $\left.\delta\right|_{A D[1 / 2,1]}=-\partial_{A D}$. The derivation $\delta$ cannot be extended up to a derivation from $S(\mathcal{R})$ to $S(\mathcal{R})$.

Proof. Let $\pi: L_{\infty}[0,1] \longrightarrow \mathcal{D}$ be a $*$-isomorphism defined in Section 5.2 .

Let $\partial_{A D}: A D[0,1] \rightarrow S[0,1]$ be the approximate derivation. By Proposition 4.13 this derivation is non-expansive. Therefore, by [5, Theorem 3.1] there exists a derivation $\delta_{0}: S[0,1] \rightarrow S[0,1]$, which extends $\partial_{A D}$.

Denote for brevity, the first Rademacher function by $r=\chi_{\left(0, \frac{1}{2}\right)}-\chi_{\left(\frac{1}{2}, 1\right)}$ and consider the mapping $\tilde{\delta}: S[0,1] \rightarrow S[0,1]$ defined by

$$
\tilde{\delta}(x)=\delta_{0}(x r), \quad x \in S[0,1] .
$$

Since $\delta_{0}(r)=\partial_{A D}\left(r^{2}\right)=0$, it follows that $\tilde{\delta}$ is a derivation on $S[0,1]$.

We set

$$
\delta=\pi \circ \tilde{\delta} \circ \pi^{-1}: S(\mathcal{D}) \longrightarrow S(\mathcal{D}) .
$$

We claim that $\delta$ is a derivation, which cannot be extended up to a derivation from $\mathcal{R}$ to $S(\mathcal{R})$. Assume, by contradiction, that the derivation $\delta$ extends up to a derivation $D: \mathcal{R} \rightarrow S(\mathcal{R})$.

Consider the automorphisms $\gamma \in \operatorname{Aut}\left(L_{\infty}[0,1]\right)$ defined by setting

$$
(\gamma(x))(t)=x(\{t+1 / 2\}), \quad x \in L_{\infty}[0,1],
$$

where $\{t\}$ is the fractional part of a number $t \in \mathbb{R}$. Since $\gamma\left(\chi_{X_{1}}\right)=1-\chi_{X_{1}}$ and $\gamma\left(\chi_{X_{k}}\right)=\chi_{X_{k}}$ for $k>1$, we obtain that

$$
\pi(\gamma(x))=u \pi(x) u, \quad x \in S[0,1],
$$

where

$$
u=\left(\begin{array}{ll}
0 & 1 \\
1 & 0
\end{array}\right) \otimes\left(\bigotimes_{i=2}^{\infty} 1_{i}\right) .
$$

Let $f \in L_{\infty}[0,1]$ be given by $f(t)=t$. Define the self-adjoint element $x_{0} \in \mathcal{D}$ by setting

$$
x_{0}=\frac{1}{4}+\pi(f \cdot r)
$$

It is clear that,

$$
\delta\left(x_{0}\right)=\pi(\tilde{\delta}(f \cdot r))=\pi\left(\delta_{0}(f)\right)=1
$$

and $\gamma\left(x_{0}\right)=-x_{0}$, because $\gamma(f)=f+\frac{1}{2} \chi_{\left(0, \frac{1}{2}\right)}-\frac{1}{2} \chi_{\left(\frac{1}{2}, 1\right)}$ and $\gamma(r)=-r$. In particular, we have $u x_{0} u=-x_{0}$ and $u x_{0}=-x_{0} u$.

By the Leibniz rule, we have

$$
D\left(u x_{0} u\right)=D(u) x_{0} u+u D\left(x_{0}\right) u+u x_{0} D(u)=D(u) x_{0} u+1+u x_{0} D(u) .
$$


Since $u x_{0} u=-x_{0}$, it follows that

$$
-1=D\left(-x_{0}\right)=-D\left(u x_{0} u\right)=D(u) x_{0} u+1+u x_{0} D(u) .
$$

Taking into account that $u x_{0}=-x_{0} u$, we obtain

$$
-2=-D(u) u x_{0}-x_{0} u D(u) .
$$

Since

$$
D(u) u+u D(u)=D(u u)=D(1)=0
$$

it follows that

$$
-2=u D(u) x_{0}-x_{0} u D(u)=\left[u D(u), x_{0}\right] .
$$

Since $x_{0}$ is self-adjoint, the latter equality contradicts Theorem 2.2 (c).

Thus, $\delta: A D(\mathcal{D}) \rightarrow S(\mathcal{D})$ cannot be extended up to a derivation from $S(\mathcal{R})$ to $S(\mathcal{R})$.

We now prove a result similar to Theorem 6.1 for an arbitrary Cartan masa in $\mathcal{R}$.

Theorem 6.2. Let $\mathcal{A}$ be a Cartan masa in the hyperfinite $I I_{1}-$ factor $\mathcal{R}$. There exists a derivation $\delta: \mathcal{A} \rightarrow S(\mathcal{A})$ which cannot be extended as a derivation from $\mathcal{R}$ to $S(\mathcal{R})$.

Proof. By Connes-Feldmann-Weiss Theorem [14, Corollary 11], there is an *automorphism $\alpha \in \operatorname{Aut}(\mathcal{R})$ such that $\alpha(\mathcal{D})=\mathcal{A}$. Since any $*$-automorphism on $\mathcal{R}$ preserves the trace, it follows that the *-automorphism $\alpha$ uniquely extends to a continuous in the measure topology $*$-automorphism of the Murray-von Neumann algebra $S(\mathcal{R})$, which we still denote by $\alpha$ (see Proposition 2.1).

Now, let $\delta: \mathcal{D} \rightarrow S(\mathcal{D})$ be the derivation as in Theorem 6.1. Then the mapping $\alpha \circ \delta \circ \alpha^{-1}: \mathcal{A} \rightarrow S(\mathcal{A})$ is well-defined and is a derivation. If $\alpha \circ \delta \circ \alpha^{-1}$ extends to a derivation $D$ from $\mathcal{R}$ into $S(\mathcal{R})$, then a derivation $\alpha^{-1} \circ D \circ \alpha: \mathcal{R} \rightarrow S(\mathcal{R})$ is an extension of $\delta$, which is not possible. Thus $\delta$ cannot be extended up to a derivation from $\mathcal{R}$ to $S(\mathcal{R})$.

\section{REFERENCES}

[1] S. Albeverio, Sh. A. Ayupov, K. K. Kudaybergenov, Structure of derivations on various algebras of measurable operators for type I von Neumann algebras, J. Funct. Anal. 256 (2009) 2917-2943.

[2] M. Argerami, P. Massey, A Schur-Horn theorem in $\mathrm{II}_{1}$ factors. (English summary) Indiana Univ. Math. J. 56 (2007), no. 5, 2051-2059.

[3] Sh. A. Ayupov, Derivations in algebras of measurable operators, Dokl. Uzbek Akad. Nauk 3 (2000), $14-17$.

[4] A. F. Ber, V. I. Chilin, F. A. Sukochev, Derivations in regular commutative algebras, Math. Notes. 75 (2004) 418-419.

[5] A.F. Ber, V.I. Chilin, F.A. Sukochev, Non-trivial derivations on commutative regular algebras, Extracta Math., 21 (2006), 107-147.

[6] A. F. Ber, V. I. Chilin, F. A. Sukochev, Continuity of derivations of algebras of locally measurable operators, Integr. Equ. Oper. Theory, 75 (2013), 527-557.

[7] A. F. Ber, V. I. Chilin, F. A. Sukochev, Continuous derivations on algebras of locally measurable operators are inner, Proc. London Math. Soc. 109 (2014) 65-89.

[8] A.F. Ber, B. de Pagter, F.A. Sukochev, Derivations in algebras of operator-valued functions, J. Operator Theory, 66 (2011), Issue 2, 261-300.

[9] A. Ber, F. Sukochev, D. Zanin, Heisenberg relation for locally measurable operators, Advances in Mathematics, vol. 335, pp. 211 - 230.

[10] O. Bratteli and D. Robinson, Operator algebras and quantum statistical mechanics, Vol. 1 SpringerVerlag, 1979.

[11] V. I. Chilin, A. V. Krygin, F. Sukochev, Local uniform and uniform convexity of non-commutative symmetric spaces of measurable operators, Math. Proc. Camb. Phil. Soc. 111 (1992), 355-368. 
[12] V. I. Chilin, F. Sukochev, Weak convergence in non-commutative symmetric spaces, J. Operator Theory 31 (1994), 35-65.

[13] L. J. Ciach, Linear-topological spaces of operators affiliated with von Neumann algebra, Bull. Polish Acad. Sc.,

[14] A. Connes, J. Feldman, B. Weiss, An amenable equivalence relation is generated by a single transformation, Ergodic Theory Dynamical Systems, 1 (1982), 431-450.

[15] M. M. Czerwińska, A. Kamińska, Geometric properties of noncommutative symmetric spaces of measurable operators and unitary matrix ideals, Comment. Math. 57 (2017), no. 1, 45-122.

[16] M. M. Czerwińska, A. Kamińska, k-Extreme Points in Symmetric Spaces of Measurable Operators, Integral Equ. Oper. Theory, 82 (2015), no. 2, 189-222.

[17] H.G. Dales, Banach algebras and automatic continuity, London Mathematical Society Monographs. Oxford University Press; (2001)

[18] J. Dixmier, Les algebres d'operateurs dans l'Espace Hilbertien, 2nd ed., Gauthier-Vallars, Paris, 1969.

[19] P. Dodds, S. Ferleger, B. de Pagter, F. Sukochev, Vilenkin systems and generalised triangular truncation operator Integr. Equ. Eper. Theory (2001) 40: 403-435. https://doi.org/10.1007/BF0119813

[20] P. Dodds, B. de Pagter, F. Sukochev, Theory of noncommutative integration, unpublished manuscript (to appear).

[21] P. Dodds, F. Sukochev Non-commutative bounded vilenkin systems, Math. Scand., vol. 87, no. 1, pp. 73-92, 2000.

[22] K. Dykema, F. Sukochev, D. Zanin, A decomposition theorem in II $I_{1}$ factors. J. Reine Angew. Math. 708 (2015), 97-114.

[23] T. Fack, H. Kosaki, Generalized s-numbers of $\tau$-measurable operators, Pacific J. Math. 123 (1986), no. 2, 269-300.

[24] H. Federer. Geometric Measure Theory. Heidelberg, New York. 1996.

[25] K. R. Goodearl, Von Neumann regular rings. Monographs and Studies in Mathematics, 4. Pitman (Advanced Publishing Program), Boston, Mass.-London, 1979. xvii+369 pp.

[26] P.R. Halmos, Measure theory, Van Nostrand Reinold, Princeton, 1950.

[27] F. Hiai, Y. Nakamura, Closed convex hulls of unitary orbits in von Neumann algebras. Trans. Amer. Math. Soc. 323 (1991), no. 1, 1-38.

[28] V. Jarnik, Sur les nombres derivés approximatifs, Fund. Math. 22 (1934), 4-16.

[29] R.V. Kadison, Z. Liu, A note on derivations of Murrayvon Neumann algebras, PNAS, vol. 111, no. 6, (2014) 20872093.

[30] R. V. Kadison, Z. Liu, A. Thom, A note on commutators in algebras of unbounded operators, arXiv:1901.10711

[31] R. Kadison, J. Ringrose, Fundamentals of the Theory of Operator Algebras I, Academic Press, Orlando, 1983.

[32] R. V. Kadison and J. R. Ringrose, Fundamentals of the Theory of Operator Algebras, vol II, Academic Press, 1986.

[33] S. Lord, F. Sukochev, D. Zanin, Singular traces. Theory and applications. De Gruyter Studies in Mathematics, vol. 46, De Gruyter, Berlin, 2013.

[34] L. Lusternik, V. Sobolev. Elements of functional analysis. Halsted Press. New York. 1974.

[35] E. Nelson, Notes on non-commutative integration, J. Funct. Anal., 15, Issue 2 (1974), 103-116.

[36] J. von Neumann, Continuous rings and their arithmetics. Proc. Nat Acad. Sci. U.S.A., 23, (1937) 341-349.

[37] J. von Neumann, The non-isomorphism of certain continuous rings, Annals of Mathematics, 67, No. 3 (1958), pp. 485-496.

[38] J. von Neumann, Continuous geometry, Princeton Landmarks in Mathematics, Princeton University Press, (1960).

[39] J. von Neumann, Collected works. Volume IV. Continuous geometry and other topics. Pergamon Press. 1962.

[40] B. de Pagter, F. Sukochev, Commutator estimates and R-flows in non-commutative operator spaces. Proc. Edinb. Math. Soc. (2) 50 (2007), no. 2, 293-324.

[41] S. Sakai, Operator algebras in dynamical systems, Cambridge University Press, 1991.

[42] S. Saks, Theory of the integral, Dover Publications, 2005.

[43] T. Scheckter, F. Sukochev, Weak type estimates for the noncommutative Vilenkin-Fourier series, Integr. Equ. Oper. Theory (2018) 90: 64. https://doi.org/10.1007/s00020-018-2489-8 
[44] I.E. Segal, A non-commutative extension of abstract integration, Ann. Math. 57 1953, 401-457.

[45] A. M. Sinclair, R. R. Smith, Finite von Neumann algebras and masas. London Mathematical Society Lecture Note Series: 351, Cambridge University Press, 2008.

[46] L.A. Skornyakov, Complemented modular lattices and regular rings, London: Oliver and Boyd 1964.

[47] S. Stratila, L. Zsido, Lectures on von Neumann Algebras. Tunbridga Wells; Kent: Abacus Press, 1979.

[48] M. Takesaki, Theory of Operator Algebras I, Springer-Verlag, New York, 1979.

[49] M. Takesaki, Theory of Operator Algebras II, Springer-Verlag, Berlin-Heidelberg-New York, 2003.

[50] M. Takesaki, Theory of Operator Algebras, III, Volume Springer-Verlag, Berlin (2003).

[51] R. J. Tauer, Maximal abelian subalgebras in finite factors of type II. Trans. Amer. Math. Soc., 114 1965, 281-308.

Department of Mathematics, National University of Uzbekistan, Vuzgorodok, 100174, TASHKENT, UZBEKISTAN

E-mail address: aber1960@mail.ru

Department of Mathematics, Karakalpak State University, Ch. Abdirov 1, Nukus 230113, UZBEKISTAN

E-mail address: karim2006@mail.ru

School of Mathematics and Statistics, University of New South Wales, Kensington, 2052, Australia

E-mail address: f.sukochev@unsw.edu.au 